\title{
"Neighborhood watch" model: embryonic epiblast cells assess positional information in relation to their neighbors
}

\section{- Short title: Neighborhood watch model}

Hyung Chul Lee ${ }^{1} \dagger$, Cato Hastings ${ }^{1} \dagger$, Nidia M.M. Oliveira ${ }^{1}$, Rubén Pérez-Carrasco ${ }^{2}$, Karen M. Page $^{3}$, Lewis Wolpert ${ }^{1} \ddagger$ and Claudio D. Stern ${ }^{1} *$

${ }^{1}$ Department of Cell and Developmental Biology, University College London, Gower Street, London WC1E 6BT, UK.

${ }^{2}$ Department of Life Sciences, Imperial College London, South Kensington Campus, London SW7 2AZ, U.K.

${ }^{3}$ Department of Mathematics, University College London, Gower Street, London WC1E 6BT, U.K.

$\uparrow$ These authors contributed equally.

\$ Deceased 28 Jan 2021

* correspondence to: $\underline{\text { c.stern@ucl.ac.uk }}$

\begin{abstract}
In many developing and regenerating systems, tissue pattern is established through gradients of informative morphogens, but we know little about how cells interpret these. Using experimental manipulation of early chick embryos including misexpression of an inducer (VG1 or ACTIVIN) and an inhibitor (BMP4), we test two alternative models for their ability to explain how the site of primitive streak formation is positioned relative to the rest of the embryo. In one model, cells read morphogen concentrations cellautonomously. In the other, cells sense changes in morphogen status relative to their neighborhood. We find that only the latter model can account for the experimental results, including some counter-intuitive predictions. This mechanism (which we name "neighborhood watch" model) illuminates the classic "French Flag Problem" and how positional information is interpreted by a sheet of cells.
\end{abstract}

\section{Teaser}

How do cells know their position in the embryo, to determine where gastrulation will start?

\section{Introduction}

In the late 1960s, Lewis Wolpert introduced the concept of "positional information", asking the question of how cells within a morphogenetic field could adopt several (more than two) cell-type identities in response to signaling cues from the embryo. The analogy of a French flag, with three colors: red, white and blue, was used to symbolize the cell types (1). Wolpert proposed that two distinct mechanisms could account for the "French flag problem". One involves a gradient of a hypothetical "morphogen" which diffuses away from a local source, decaying with distance; this would be read by cells, which 
respond with discrete thresholds to adopt the various identities. In a later paper (2), Wolpert suggested that intercellular communication is required for cells to assess their position with respect to each end of the field. The second mechanism ("balancing model") involves a process by which cells compare themselves to their immediate neighbors to set the boundaries between territories in a more dynamic way. Both models could account for many developmental situations, but it is mainly the first model (morphogen gradient with thresholds) that has become generally associated with the problem of how positional information is encoded; a recent review summarizes the conceptual history of this (3). The proposal that intercellular communication is required to interpret these gradients has often been overlooked in more recent descriptions of the "French Flag model".

Strong support for the idea of a morphogen gradient with multiple thresholds arose from findings in which a mesoderm inducing substance ("XTC-MIF", now known to correspond to a VG1/ACTIVIN/NODAL-like member of the TGF $\beta$ family of proteins) can induce different types of mesoderm from Xenopus ectoderm in a graded way: very small differences in concentration (with variation as fine as $1.5 \mathrm{x}$ ) specify unique cell types (4). In other systems, evidence for long-range gradients of morphogens that specify different states has also been obtained; probably the best example of this is the cnidarian Hydra where there appear to be several such morphogens: activators and inhibitors of "head" formation and activators and inhibitors of "foot" development. The relative concentrations of these determine whether the head (mouth and tentacles) or foot are regenerated from body fragments $(5,6)$. These examples, as well as the simplicity of the idea of gradients of diffusible morphogens interpreted with distinct thresholds, probably account for the greater popularity of this model to explain the "French flag problem". However, few studies have asked this explicitly in other systems.

Here we use the very early chick embryo, at the time the primitive streak arises to break the initial radial symmetry of the flat blastodisc (establishing bilateral symmetry of the future embryo), to study how cells acquire positional information. In these early embryos, the "pattern" is established in the marginal zone, a ring-like region of extraembryonic tissue, lying just outside of the central disk-like area pellucida, where the embryo will arise. The primitive streak, the first indication of the future midline of the embryo, arises at one edge of the inner area pellucida, adjacent to the posterior part of the marginal zone, where the TGF $\beta$-related signaling molecule cVG1 (currently designated GDF3 in gGal6 but more closely homologous and syntenic to mammalian GDF1) is expressed. Previous studies have shown that positioning of the primitive streak requires "positive" inducing signals by cVG1/NODAL from the posterior marginal zone near the site of streak formation, and that this is antagonized by BMP signaling which is highest at the opposite (anterior) end of the blastoderm (fig. S1A) (7-13).

The ring-like marginal zone of these early embryos allows a study of "pattern formation" in a one-dimensional system, similar to the "French Flag Problem". To find out how cells interpret morphogen concentrations to generate positional information, we designed two computational models to represent respectively a fixed gradient, read locally by cells, or a system where cells compare themselves to their neighbors to determine their position in 
the field. Using a combination of embryological manipulations and computational modelling, we ask which of these two models can best account for the results of various manipulations in the spatial distribution, number and intensity of the inducing (cVG1/NODAL) and inhibitory (BMP) signals. Our results suggest that the "positional information" that determines the site of primitive streak formation is explained better by a mechanism by which cells compare themselves to their neighbors rather than by a cell autonomous assessment of gradients. We name this the "neighborhood watch" model.

\section{Results}

\section{Epiblast cells may sense local differences in strength of inducing signal rather than the absolute amount of inducer}

When a small pellet of cVG1-expressing cells (HEK293T cells transfected with a cVG1expression construct) is grafted into the anterior marginal zone (the innermost extraembryonic epiblast, just outside the central embryonic area pellucida), it can initiate formation of an ectopic primitive streak that eventually develops into a full embryonic axis $(7,12)$. However endogenous $c V G 1$ mRNA is expressed as a crescent encompassing an arc of about $60^{\circ}$ in the posterior marginal zone (fig. S1A). To mimic this distribution more closely, as well as to test the effects of greater concentration of cVG1 inducing signal, we placed two cVG1-expressing cell pellets side-by-side in the anterior marginal zone, and assessed primitive streak formation by in situ hybridization for expression of $B R A C H Y U R Y(c B R A,=T B X T)$ after overnight culture (Fig. 1 A-D). Only a single ectopic primitive streak was generated near the middle of the two cVG1 pellets (Fig. 1 B); neither double nor thicker ectopic streaks were observed, similar to the effects of implanting a single pellet.

To provide a stronger and wider signal, we tested the effect of implanting four cVG1expressing cell pellets side-by-side in the anterior marginal zone. Surprisingly, in the majority of cases (11/16 embryos), no ectopic primitive streak formed and no ectopic $c B R A$ expression was seen (Fig. $1 \mathrm{E}, \mathrm{H}, \mathrm{K}$ ). Since application of the equivalent of a quaddose of inducer spread over a four-fold wider area does not cause either more efficient or wider induction than a single dose, we speculated that "boundaries" to the signaling domain may be required. To test this, we placed a control cell pellet (HEK293T cells transfected with pCA $\beta$-GFP; see Methods) to split four cVG1-expressing cell pellets into two groups on either side. The incidence of ectopic streak formation doubled (Fig. $1 \mathrm{~F}, \mathrm{I}$, $\mathrm{K})$. If a boundary is indeed important, we might expect that, perhaps paradoxically, ectopic streak induction might increase if a pellet expressing the inhibitor BMP4 (rather than a control pellet) is used to interrupt the set of four cVG1-expressing cell pellets. This is indeed the case (Fig. $1 \mathrm{G}, \mathrm{J}, \mathrm{K}$ ). Together, these results suggest that cells may sense variations in signal strength in relation to their neighbors, rather than measuring the absolute amount of local signal they receive, to determine the outcome of the inductive event.

The above experiments were done using pellets of transfected cells, as in previous studies $(7-9,12-14)$. One problem with this approach is that cells are likely to express other 
(unknown) factors that could influence the outcome of the signaling event. Another problem is that these pellets are relatively large (500-1000 cells). We therefore decided to substitute the use of cell pellets with protein-soaked microbeads (about $100 \mu \mathrm{m}$ diameter). As neither VG1 nor NODAL are available as pure proteins, we decided to use ACTIVIN instead, which can induce axial structures and mesendodermal markers in chick epiblast $(15,16)$. As shown in amphibian animal cap ectoderm explants (4), ACTIVIN also acts through the SMAD 2/3 pathway and generates finely graded responses of mesendoderm induction to different concentrations (16). BMP4-soaked beads were used as a source of inhibitory signal. First, we checked if a single soaked bead can mimic the effects of a single cell pellet (fig. S2). Grafting a bead soaked in ACTIVIN into the anterior marginal zone has the same effect as a cell pellet placed in the same position: it induces an ectopic $c B R A$-expressing primitive streak in adjacent epiblast (fig. $\mathrm{S} 2, \mathrm{~A}-\mathrm{E})$. Conversely, placing a bead of the inhibitor BMP4 in the posterior marginal zone results in either displacement of the endogenous primitive streak to a more lateral position, or two primitive streaks, arising either side of the BMP4-bead (fig. S2, F-J). With a high concentration of BMP4 $(50 \mathrm{ng} / \mu \mathrm{l})$ primitive streak formation was inhibited in about half of the embryos (fig. S2J).

Next, we mirrored the experiments done with two or more cell pellets but using soaked beads (Fig. 2). When three ACTIVIN protein-soaked beads (ACTIVIN bead) were grafted in a row to expose a wide domain to the inducing signal, the majority of embryos $(78 \%, 7 / 9)$ showed no ectopic $c B R A$ expression (Fig. 2, B, F, I). When boundaries to the signaling domain were generated either by introducing a BSA-soaked control bead (Fig.2, C and G) or a BMP4-soaked bead (Fig. 2, D and H) among the ACTIVIN beads, the proportion of embryos with ectopic $c B R A$ expression increased to $40 \%$ (4/10) and $50 \%(6 / 12)$, respectively (Fig. 2 I). Therefore, as with experiments using cell pellets, these results suggest that cells may sense inducing signals relative to their neighbors, rather than the absolute local amount of inducing signal.

\section{Two alternative models}

To distinguish between the two alternative mechanisms of how cells might sense their positions (absolute local morphogen concentration or comparison of local signal strength in relation to their neighborhood), two mathematical models were designed, one for each of these mechanisms, to make experimentally-testable predictions (for details see Materials and Methods). We model the marginal zone as a one-dimensional ring of cells (Fig. 3 A). Positional information is provided by the balance between an inducer (SMAD2/3 activation in response to a VG1/ACTIVIN/NODAL-type signal) and an inhibitor (SMAD 1/5/8 in response to a BMP signal) within each cell (Fig. 3 B). Model A proposes that each cell independently assesses the concentration of morphogens (inducer vs. inhibitor) it receives: when a threshold is exceeded, the cell is triggered to start primitive streak formation. Model B proposes that cells communicate with their neighbors to assess how the streak-inducing signal changes in space: each cell in the ring compares itself with the average signal strength in its neighborhood to determine whether or not to initiate streak formation (Fig. 3 B). 
As an initial test of the model, we used Bayesian parameter estimation to infer distributions of parameters with associated scores assessing how well both models replicate the results shown in Fig. 2. The score was calculated using a 'likelihood function' (fig. S3) which quantifies how well the predicted number and position of ectopic streaks match experimental results on a cell-by-cell basis. As an additional verification, the number and position of ectopic primitive streak formation was also assessed qualitatively to define the 'success' of model predictions. Parameter values were chosen to maximize the model success rate and the likelihood score. Fig. 3 C-G summarize the results of these simulations. No set of parameter values was found that allowed Model A to replicate both experimental results, when either one or three beads of inducer were placed in the anterior marginal zone (Fig. 3 C, D). In contrast, Model B successfully predicted that broadening the domain of ectopic inducer reduces the chance of initiating ectopic streak formation (Fig. 3 D).

The two models also differ in their ability to portray the effects of placing a bead of inhibitor between two beads of inducer (Fig. 3 E-G). Model A predicts that the presence of the inhibitor will reduce the likelihood of ectopic streaks (Fig. 3 E, F). However, Model B correctly predicts that a low dose of inhibitor increases the chance of forming an ectopic streak (Fig. $3 \mathrm{G}$ ). The same results were obtained irrespective of whether the sources of inducer and inhibitor were of small diameter (Fig. $3 \mathrm{C}-\mathrm{G}$, to simulate microbeads as in Fig. 2) or wider (fig. S4, simulating a cell pellet as in Fig. 1).

We sought a single set of bead parameters that would allow both models to mimic the experimental findings (Fig. $3 \mathrm{H}$ ). However, choosing a single set of bead parameters could act as a constraint, giving an advantage to one of the models. Therefore, we also performed the parameter inference to allow bead parameters to vary for each model independently (Fig 3 I). Strikingly, Model B always outperforms Model A, regardless of whether a single set of parameters is chosen to fit both models, or whether parameter values are optimized for each model separately (Fig. $3 \mathrm{~J}$ ).

\section{Challenging the models and testing predictions}

\section{a. Decreasing the amount of inhibitor}

In both models, cells measure their position by assessing the relative strength of the intracellular downstream effectors of the inducers (VG1/NODAL/ACTIVIN) and inhibitors (BMP). Therefore, decreasing the streak-inhibiting signal alone should induce ectopic primitive streak formation. In this case, both models predict this outcome (Fig. 4 A and B).

To test these predictions experimentally, we used dorsomorphin, an inhibitor of BMP signaling (17). A dorsomorphin-soaked bead was grafted in the anterior marginal zone (Fig. 4 A). After overnight culture, an ectopic primitive streak (with $c B R A$ expression) was seen to arise close to the bead (Fig. $4 \mathrm{C}$ and D). This result is consistent with a previous study showing that a graft of a cell pellet expressing the BMP antagonist CHORDIN in the area pellucida induces an ectopic streak (8). When embryos that had 
been grafted with a dorsomorphin-bead were examined 6 hours after the graft, ectopic expression of $c V G 1 \mathrm{mRNA}$ in the area pellucida ( $c V G 1$ expression is an early target of VG1/NODAL signaling; $(12,14)$ ) was found in the vicinity of the bead (Fig. 4 E and F).

\section{b. Increasing the amount of inhibitor}

A more counterintuitive prediction arises when the strength of inhibition by BMP is increased in a region that normally expresses high levels of BMP (Fig. 5 A). The two models predict different outcomes: Model A predicts that increasing BMP signaling in the anterior marginal zone will reduce the chance of ectopic streak formation (Fig. $5 \mathrm{~B}$ ). Counterintuitively however, Model B predicts that introducing a bead of inhibitor will increase the streak-inducing values in an area adjacent to the bead (bottom, Fig. 5 B). However Model B also suggests that this effect will be small, perhaps insufficient to result in formation of a mature ectopic primitive streak.

In embryological experiments in which a BMP4 bead was grafted into the anterior marginal zone, no $c B R A$ expression or streak formation was observed after overnight incubation (Fig. 5 C). After short incubation (4.5 h), however, $c V G 1$ expression was observed in cells surrounding the grafted BMP4 bead in the anterior marginal zone and slightly in the adjacent area pellucida (Fig. 5 D). $c V G 1$-expression was absent from cells directly overlying the bead (Fig. 5 F) (see also (18)). In addition, the ectopic expression was very weak, only detectable after prolonged chromogenic development of the in situ hybridization (Fig. $5 \mathrm{D}$ and F). This ectopic expression of $c V G 1$ in the anterior marginal zone was transient: it was seen at $4.5 \mathrm{~h}$ and disappeared by $6 \mathrm{~h}$, remaining mostly in the lower layer of the area opaca (germ wall; Fig. $5 \mathrm{E}$ and $\mathrm{G}$ ). In conclusion, this experimental result conforms with the predictions of Model B but not those of Model A.

\section{c. Effect of adjacent sub-threshold amounts of inducer and inhibitor}

We have seen that an increase in streak-inhibiting signal can result in paradoxical induction of $c V G 1$, which is only predicted by Model B. However, no ectopic $c B R A$ expression is observed. If it is indeed the case that cells assess their position in comparison with their neighbors (Model B), rather than measuring the absolute local levels of inducer and inhibitor, then introducing a sub-threshold amount of inducer flanked by low amounts of inhibitor would both deepen and steepen the gradient and might therefore be expected, perhaps paradoxically, to generate a new streak. Model A, in contrast, might predict that neither concentration is high enough locally to affect cell fates resulting in a failure of ectopic streak formation. To simulate this, we explored parameter values for both models that could generate this result (Fig. 6). We find that only Model B can predict the initiation of an ectopic streak (Fig. 6 D-F). No parameters were found that allowed Model A to produce the same result (Fig. 6 D-F).

Next, we tested this prediction experimentally. We began by establishing the minimum threshold of ACTIVIN concentration for PS induction; $2.5 \mathrm{ng} / \mu \mathrm{l}$ of ACTIVIN does not induce cBRA (fig. S2 D). When two BMP4-beads $(6.25 \mathrm{ng} / \mu \mathrm{l})$ were separated by a control bead, no ectopic PS formed (Fig. 6 A and G). When an ACTIVIN-bead (2.5 
$\mathrm{ng} / \mu \mathrm{l})$ was flanked by control beads, $94 \%$ of embryos showed no ectopic primitive streak $(\mathrm{n}=17)$ (Fig. $6 \mathrm{~B}$ and $\mathrm{H}$ ). We then tested the predictions of the model experimentally: when a sub-threshold ACTIVIN bead was flanked by BMP4 beads, $c B R A$ expression was seen in $23 \%$ of cases $(n=13)$ (Fig. $6 \mathrm{C}$ and I). However, a higher concentration of BMP4 $(12.5 \mathrm{ng} / \mu \mathrm{l})$ in the neighboring beads reduced the proportion of embryos with an ectopic streak (to 9\%; $n=22$ ) (data not shown), suggesting that at this concentration the total amount of inhibitor may overcome the small amount of inducer emitted by the subthreshold ACTIVIN-bead. In conclusion, therefore, only Model B correctly predicts the counterintuitive results of this experiment.

Taken together (Fig. 7) our results strongly favor a model by which cells assess their status (in terms of whether or not they will constitute a primitive-streak-initiating center) in relation to the relative amounts of inducing and inhibiting signals they experience and also in relation to the status of their neighbors, rather than by direct readout of the local concentration of a morphogen that diffuses freely across the entire embryo.

\section{Discussion}

When the 'French Flag problem' was first proposed, two alternative mechanisms that could generate a pattern of territories within a one-dimensional (linear) field were suggested. One relies on a "fixed gradient": "a unit can compute exactly where it is in the system and can thus 'decide' what state it should be in. A simple mechanism whereby this can be provided is by having two accurate gradients generated from the two ends of the system. The unit can compute its position from the value of the two gradients and so enter into the appropriate state." (1). The example developed more fully in the subsequent discussion relies on just one gradient of a single substance, "I", which freely diffuses across the field from left to right, whose concentration is read locally by cells in the field. The property encoding the position of a cell in the field was called "positional information" (2). The second model was called the "balancing" model: "the unit cannot compute where it is in the system in relation to the two ends. The mechanism relies of 'voting' or 'balancing' of the sizes of the regions against each other' (1). This model proposes that regions (like the red, white and blue of the French flag) are formed of units (i.e. cells) in the same state, and the regions are separated by distinct boundaries. Each cell emits signals to induce changes in neighboring cells to states different from the emitting cell. As these substances rapidly diffuse and decay, cells change state until the signal concentrations balance out at the boundaries between regions, fixing their relative sizes. The balancing mechanism relies crucially on the rates of production, diffusion and decay of the putative signaling molecules. Although even the "fixed gradient" model proposed that intercellular communication may be required for the interpretation of positional information (2), the significance of this has been lost in later discussions of the 'French Flag model', which is now synonymous with cell-autonomous sensing of the local concentration of a morphogen that is distributed as a longer-range gradient.

Here, we propose that each cell measures the relative strength of inhibitory (BMP) and inducing (cVG1/NODAL) signals it receives. One likely mechanism for this is that the comparison involves competition of SMAD1/5/8 (effectors of BMP signaling) and SMAD2/3 (effectors of VG1/ACTIVIN/NODAL) for binding to SMAD4 (called "co- 
SMAD"), if the concentration of the latter is limiting. Our model uses this "SMADvalue" to represent this, and indirectly therefore the position of the cell within the field. This mechanism does not require very long-range diffusion but still retains the main features of the "fixed gradient" model.

Do gradients exist, and over what distance can they provide positional information? In the chick embryo, two opposing gradients are detectable by mRNA in situ hybridization or RNA-sequencing $(7,8,19)$ : a gradient of the inhibitor BMP4 (highest anteriorly), and a region of high expression of the inducer $c V G 1$ posteriorly, which rapidly decreases anteriorly. Could these gradients be generated and/or maintained by free diffusion of these ligands over the entire marginal zone? The chick marginal zone spans a distance of about $3 \mathrm{~mm}$ between the anterior and posterior extremes, and comprises a single sheet of epithelial cells connected by intercellular junctions. The whole embryo is a thin sheet of epiblast, suspended at the interface between two liquids: the yolk (below) and the albumen (dorsally). Each cell of the marginal zone is quite small, about $15 \mu \mathrm{m}$ in diameter. Therefore, the anterior-posterior distance between the two extremes of this ring should span about 300 cell lengths (in reality the marginal zone has a thickness of about $120 \mu \mathrm{m}$, corresponding to about 10 cells - here we represent it as being one-cell-thick). It seems unlikely that this geometry can support the formation or maintenance of longrange gradients of morphogens generated by free diffusion. Indeed, Crick (20) calculated that such gradients cannot span more than about $1 \mathrm{~mm}$, and are more likely to be restricted to around 50-70 cell diameters. Most likely, therefore, these gradients do not involve free, unrestricted diffusion in the extracellular space, but are somehow maintained by cell interactions across the large field of cells. In the chick embryo, $c V G 1$ mRNA expression is restricted to a fairly narrow arc at the posterior end of the marginal zone, and BMP4 expression appears as a shallower gradient, highest anteriorly and decreasing posteriorly quite gradually along the periphery of area opaca and marginal zone. This expression profile might indeed generate gradients of the two proteins, but not by free diffusion beyond a few cell diameters; these gradients are then interpreted by the responding cells based on the "SMAD-value" whereby cells compute their position in the field.

Therefore, in our system, the "SMAD-value" could be seen as a key component of "positional information". However, our present results, both from computational modeling and experiments, strongly favor the idea that cells do not read the concentrations of inducer and inhibitor ("SMAD-value") locally and cell autonomously, but rather interpret their own SMAD-value in relation to that of their neighbors. Moreover, the results also suggest that the distance over which such comparisons take place is greater than just the immediately neighboring cell on either side.

How do cells communicate with their neighbors? In other words, by what mechanism could cells assess their environment? In the wing imaginal disc of Drosophila embryos, the TGF $\beta$-related protein Decapentaplegic (Dpp) acts as a morphogen conveying positional information that results in positioning the wing veins and other features of the wing. Signal-receiving cells have been shown to extend thin and long filopodia, called cytonemes, which extend several cell diameters to the proximity of Dpp-producing cells (21-23). It is worth noting that the existence of very long filopodia extending very large distances (connecting the invaginating archenteron with the future oral ectoderm at the opposite end of the embryo) was observed by Gustafson and Wolpert in studies of 
gastrulation in the sea urchin in 1961 (24) - this was one of the studies that initiated thinking on pattern formation. As yet, these structures have not been observed in early chick embryos, but this has not yet been investigated in detail.

Is our present model closer to the "balancing" model (1)? This model requires that the system initially has two states, and that these are separated by a clear boundary. This does not appear to be the case in the early chick and we have not assumed this situation in our model. In addition, the "balancing model" proposes that changes in the state of a unit (or cell) can only occur at boundaries between states. This is also not the case in our model. Finally, it also implies that all cells are held poised, with only local cell-cell inhibition preventing a change in state. Again, we have not incorporated this feature in the model and it does not appear to be borne out by the patterns of expression seen in the embryo. In fact, rather than sensing a boundary between adjacent regions of different state, our model proposes that cells sense different levels of signal (SMAD-value) relative to their neighbors, which can be achieved by varying the levels of either the inducer, or the inhibitor, or both.

Both the "balancing" and the "fixed gradient" models were proposed to explain size regulation of the entire field: why red, white and blue territories of the French flag are of equal sizes, and they scale up or down for smaller or larger flags. In our system there does not appear to be significant size regulation of many territories, but rather the positioning of a single territory (the site that will initiate primitive streak formation) within a large field. Despite this, positioning the site of primitive streak formation within a large field and thereby establishing the polarity of that field is an example of "pattern formation". Thus, our model does not involve long-range comparisons between large, or distant territories, but rather local cell interactions between cells and their neighbors.

One question is whether the mechanism proposed here (involving only local cell interactions and no long-range diffusion) is a feature unique to very large fields (several $\mathrm{mm}$ ), where meaningful positional information conveyed only by diffusion would be impossible. There do appear to be several instances where diffusion of informative morphogens is key, such as initial patterning of the Drosophila blastoderm $(25,26)$ and mesoderm induction by activin in Xenopus animal caps (27-29). It therefore seems likely that positional information can be imparted by a variety of different mechanisms, perhaps according to the size and characteristics of the field to be patterned. It will be interesting to perform experiments comparable to those in this paper in a system such as anteriorposterior patterning of the chick limb, which is also very large and involves a localized signaling region (the Zone of Polarizing Activity) $(30,31)$.

\section{Downstream of primitive streak initiation}

Here we propose that positional information (when interpreted by a collection of cells) defines the location of the signaling center that initiates primitive streak formation. Initiation of a streak can be seen as the event which defines embryonic polarity. Our experiments and the associated models were designed to ask questions about how cells within the marginal zone assess their positions around the circumference of this signaling region, and thereafter determine the site next to which (in the area pellucida) the primitive streak will start to form. However, it is important to realize that in the embryo, 
the downstream consequence of these processes is not only a spot of $c B R A$ expression, but rather a true "streak", gradually extending towards the center of the embryo. It has been shown previously that this elongation involves a process of cell polarization and intercalation affecting the same site in the area pellucida where cells receive the inducing signals from the marginal zone (and which itself expresses cVG1 and NODAL) (32-34). Here, we observe cases where $c B R A$ is induced but this is not followed by formation of an elongated primitive streak. For example, this result is seen when three beads are placed in the anterior marginal zone (A-B-A). One possible reason for this is that the embryos were not incubated for long enough to allow the intercalation to take place, but it is also possible that signals other than $\mathrm{CVG1}$ and inhibition of BMP are required. Indeed it appears that non-canonical (planar cell polarity) WNT signaling drives intercalation (33) within the area pellucida. Whatever mechanisms operate in the normal embryo to determine the site of primitive streak formation must somehow coordinate these signaling events to generate the full structure.

It is also clear that there are more inhibitory events than the initial extraembryonic BMP signals: for example, the hypoblast emits the NODAL inhibitor Cerberus/CERL1 (11) which appears to serve the function of delaying primitive streak formation until cell intercalation has taken place in the NODAL expressing domain (34). It has also been proposed that the primitive streak itself emits inhibitors (perhaps including another NODAL antagonist, Lefty) to prevent the formation of an ectopic primitive streak close to the forming one $(11,34,35)$. The existence of several overlapping inhibitory mechanisms may explain why in some embryos, the endogenous primitive streak fails to form when an ectopic streak is induced, whereas in other cases both the endogenous and an induced streak appear. We have not attempted to model these features in our model, which is specifically designed to assess how cells measure their position within the marginal zone to determine the location of the domain that will initiate primitive streak formation.

\section{General considerations about models in biology}

Models designed to mimic biological processes are often tailored to maximize the "robustness" of their results. In the present model, a small change in parameter values can result in large changes in the type of result obtained (either formation of an ectopic streak or failure of this). This implies that the model is not robust to small changes; however, nor are the experimental results for this type of experiment, which often show a range of outcomes for each experiment, reflecting some inherent variability and/or instability of the biological system. Therefore, we propose that a more nuanced theory of robustness is required, in which models are rewarded for displaying biologically realistic variability.

Most models of biological processes are presented as a single hypothesis and designed to replicate a particular set of experimental results. One problem with this is that it is relatively straightforward to modify virtually any model appropriately so that it gives a particular outcome in a reproducible way. There are only a few examples where different models are compared in their ability to fit experimental results - a particularly successful case is the study by Sharpe and colleagues (36) on somite formation, where Bayesian methods are used to select between alternative classes of mechanism. Here we have 
followed this principle and compare two alternative models directly in their ability to fit a set of experimental results and to make counter-intuitive predictions. First, we propose one simple model by which cells measure the concentrations of inducing and inhibitory signals directly and cell-autonomously to determine their behavior, and a second simple model by which cells measure the relative change in inducer/inhibitor strength in comparison to their neighborhood (a few cell diameters); we then make the two models compete to see which one explains the phenomenology better. Then, we allow each model to determine the best parameters to increase the chances that it will generate the observed experimental result(s). Next, we repeat the comparison and ask whether in the best possible scenario, do both models perform equally well or does one win out. Finally, we use the two models to make counter-intuitive predictions, and these are tested experimentally. This unbiased approach strongly favors the second model by which cells assess their position (and therefore whether or not they will act as a primitive streak initiating center) relative to the status of their neighbors.

Taken together, we provide evidence that in a large system with two opposing gradients, cells assess their position in the field by measuring their location based on the relative concentrations of the inducing (cVG1/NODAL) and inhibitory (BMP) signals, and this is refined by taking cues from their local environment to assess the rate of change of these signals locally. However, the gradients are unlikely to involve long-range diffusion of two morphogens. Regulation of their strength is likely to involve other mechanisms resulting in gradients of transcription and therefore rates of production of the factors.

\section{Materials and Methods}

Embryo culture and wholemount in situ hybridization

Fertilized White Leghorn hens' eggs (Henry Stewart, UK) were incubated for 2-4 hours to obtain EGK X-XI embryos, which were then harvested in Pannett-Compton saline (37). After setting up for modified New culture $(38,39)$, the cell pellets or beads were grafted as required for each experiment, and the embryos cultured for the desired length of time before fixation in formaldehyde. Whole mount in situ hybridization was conducted as previously described $(40,41)$. The probes used were: $c V G 1(7), c B R A(42)$ and BMP4 (43). Stained embryos were imaged under an Olympus SZH10 stereomicroscope with a QImaging Retiga 2000R camera. Some embryos were sectioned in sectioning at $10 \mu \mathrm{m}$.

\section{Misexpression of proteins with transfected cell pellets}

HEK293T cells were seeded at $5 \times 10^{5}$ cells/well in a 6-well dish and incubated for two days (or $1 \times 10^{6}$ cells/well for transfection on the next day) at $37^{\circ} \mathrm{C}$ in a total of $2 \mathrm{ml} 10 \%$ FBS DMEM (growth medium)/well. On the day of transfection, the growth medium was changed to $1 \mathrm{ml} /$ well of 5\% FBS DMEM (transfection medium) at least $30 \mathrm{~min}$ before transfection. Transfection was carried out using PEI as reported previously (44). Briefly, $3 \mu \mathrm{l}$ PEI $(1 \mathrm{mg} / \mathrm{ml})$ was added for every $1 \mu \mathrm{g}$ of DNA transfected, in a total volume of 150 (for $0.5-2 \mu \mathrm{g}$ )-200 $\mu \mathrm{l}$ (for 3-6 $\mu \mathrm{g}$ ) DMEM in a sterile Eppendorf. $2 \mu \mathrm{g}$ DNA were transfected/well (containing $6 \mu \mathrm{l} \mathrm{PEI/well).} \mathrm{Expression} \mathrm{plasmids} \mathrm{were} \mathrm{the} \mathrm{previously}$ described DMVg1 (myc-tagged chimeric Vg1 containing the pro-domain of Dorsalin; (7), pMT23 (murine BMP4; (45), and pCA $\beta$-IRES-GFP (as a control). The latter was also used to estimate transfection efficiency. Transfection mixtures were vortexed and then 
left for 10 minutes at room temperature for the PEI/DNA to complex. The transfection mixture was then added dropwise to the confluent monolayers of cells and incubated overnight at $37^{\circ} \mathrm{C}$. The next day cells were checked for transfection efficiency of the GFP plasmid; typically, efficiency ranged from 60-90\%. Cells were washed three times with 1 $\mathrm{X}$ PBS, trypsinized and resuspended in a total of $1.5 \mathrm{ml}$ growth medium and put into a sterile Eppendorf. The cell concentration was estimated in a hemocytometer. A bulk cell suspension of the transfected cells was made in the growth medium, so that each drop contained 500 cells in a total of $20 \mu 1$ growth medium. Hanging drops were formed by placing the $20 \mu \mathrm{l}$ aliquots on the lid of a $6 \mathrm{~cm}$ cell culture dish, the bottom of which was filled with $5 \mathrm{ml}$ of sterile PBS or water to create a humidified atmosphere. After placing several such aliquots well-spaced in a circle, the lid was inverted and placed over the bottom of the dish, creating a mini culture chamber, to allow the cells to coalesce into pellets without adhering to the plastic. Culture dishes were incubated for $36-48 \mathrm{~h}$ at $37^{\circ} \mathrm{C}$ for the formation of pellets ranging in size from 500-1000 cells and used for grafts as required.

\section{Protein or chemical soaked microbeads}

Recombinant human BMP4 (R\&D systems, 312-BP) was delivered using Affigel Blue beads (BIO-RAD 1537302); recombinant human ACTIVIN A (R\&D systems; 338-AC) was delivered using Heparin-Acrylic beads (Sigma-Aldrich, H5236) and Dorsomorphin hydrochloride (Tocris 3093) was loaded onto AG1X2-formate beads. In each case the beads were incubated overnight at $4{ }^{\circ} \mathrm{C}$ in the desired concentration of protein or chemical. Beads were washed in Pannett-Compton saline just before use.

\section{Encoding the biological problem mathematically}

The marginal zone is modeled as a one-dimensional ring of cells, comprising 600 cells in total (based on the assumption that the embryo at this stage contains 20,000-50,000 cells (9) and on electron microscopy data $(19,33)$ for estimates of cell size and the radius of the marginal zone). Inducer and inhibitor concentrations are assigned to each cell, represented as $V_{i}$ and $B_{i}$ respectively in cell $i$, with $i=1, \ldots, 600$ (Fig. 3A).

Before the addition of beads, inducer and inhibitor levels are inferred from a combination of RNAseq reads (19) and in situ hybridization of $c V G 1$ and $c B M P 4$ (fig. S1A) respectively, at approximately stage EG\&K XII. The modeled levels of inducer and inhibitor are assumed to be some combination of all primitive streak-inducing and inhibiting elements. Inducer levels are modeled using a gaussian function and inhibitor levels with a parabolic function (fig. S1B).

The presence of a bead is modeled as having an additive (or subtractive) effect on local protein concentration. The added values are constant for the width of the bead, and then decrease exponentially in space. Therefore, placement of a bead invokes 4 parameters (fig. S4 A): the position of the center of the bead, the width of the bead, the bead's concentration (relating to magnitude of the added values, see fig. S4 B) and the rate of decay of the added compound in space (i.e. the 'spread' parameter of the exponential distribution, see fig. S4 C). 


\section{Defining two models}

For each cell to make its decision to initiate streak formation, we define the relationship between the amounts of SMAD2/3 (as a proxy for amount of inducer received) and SMAD1/5/8 (as a proxy for amount of inhibitor received) within the cells. This is based on the fact that inducing TGF $\beta$-related signals (VG1/ACTIVIN/NODAL) act by phosphorylation of SMAD2/3, whereas inhibitory TGF $\beta$-related signals (BMPs) phosphorylate SMAD1/5/8 - cells have been proposed to evaluate the relative strength of signals through competition of binding of these two classes of SMADs to the "co-SMAD", SMAD4 (46). Inducing and inhibitory SMADs compete to form complexes with a fixed, limited amount of SMAD4. The inducer- and inhibitor-linked SMAD complexes then move to the nucleus and regulate expression of different target genes. We define

$$
F_{i}=\frac{a_{V} V_{i}}{1+a_{V} V_{i}+a_{B} B_{i}},
$$

where $a_{V}$ and $a_{B}$ are scalings of the protein concentrations. $\left(1+a_{V} V_{\mathrm{i}}+a_{B} B_{i}\right)$ can be said to represent the total amount SMAD4 in the cell, so that $F_{i}$ represents the proportion of streak-inducing SMAD complex. $F_{i}$ will hereafter be referred to as the "SMAD-value", with higher values indicating stronger induction.

We define two models for how cells interpret the SMAD-value to make the decision to initiate a primitive streak.

A. Each cell compares its SMAD-values with a fixed threshold, without reference to its neighbors. If the threshold is exceeded, the cell is defined to take part in primitive streak initiation and will express $c B R A$. For each cell $i$, if

$$
F_{i}>\alpha
$$

then that cell forms part of the primitive streak initiating domain.

B. Each cell compares its own SMAD-value with those of its neighbors. Each cell can sense these values a certain distance away from itself and calculates an average SMAD-value for all the neighbors it can see. If its own value is sufficiently large compared to the average of its neighbors, the cell becomes part of a primitive streak initiating center, and expresses $c B R A$. Therefore, a streak is initiated next to cell $i$ if

$$
\frac{F_{i}-F_{\langle\mathrm{nbhd}\rangle}}{F_{i}}>\beta,
$$

where $F_{\langle\text {nbhd }\rangle}$ is defined to be the average value of $F_{j}$ in a given neighborhood surrounding cell $i$. Specifically,

$$
F_{\langle\text {nbhd }\rangle}=\frac{\sum_{j} F_{j}}{2 n},
$$

with $j \in[i-n, i+n] \backslash\{i\}$, where $(2 n+1)$ is the full width of the neighborhood.

Both Models A and B have as parameters a threshold value ( $\alpha$ or $\beta$ ) and protein concentration scalings $\left(a_{V}\right.$ and $\left.a_{B}\right)$. Additionally, Model B requires the size of the neighborhood $(n)$ to be defined as a parameter.

Parameter inference 
For the final stage of the modeling process, we ask whether there exists a set of parameters allowing each model to replicate a target result. Parameter values are scored using a likelihood function which quantifies how well model predictions match a target result. The target result is defined based upon an experimental result (Fig. 3 and fig. S4) or a new possible theory (Figs. 4-6).

For the parameter search, we fix the expected width of the streak initiating domain, as well as the positions and widths of the beads. We allow the concentration and spread parameters of the beads to vary (denoted $c$ and $s$ ) in addition to all model parameters ( $\alpha$, $\left.\beta, a_{V}, a_{B}, n\right)$. Uniform prior distributions are defined for all parameters except the protein concentration scalings, $a_{V}$ and $a_{B}$. For these parameters we define $b_{V}=\log _{10} a_{V}$ and $b_{B}=\log _{10} a_{B}$, which are then uniformly distributed. We define biologically plausible ranges within which parameters are allowed to vary (shown in fig. S6).

In order to obtain the likelihood function, we first define for each cell, the distance $\left(f_{i}\right)$ of the SMAD-value $\left(F_{i}\right)$ to the threshold for streak formation, which for Model A is

and for Model B

$$
f_{i}^{(\mathrm{A})}=F_{i}-\alpha
$$

$$
f_{i}^{(\mathrm{B})}=\frac{F_{i}-F_{\langle\mathrm{nbhd}\rangle}}{F_{i}}-\beta .
$$

So $f_{i}>0$ implies that a streak will form in cell $i$, and $f_{i} \leq 0$ implies no streak will form. For convenience we can write that $f_{i}=f_{i}(\theta)$ where $\theta=\left\{\alpha, \beta, a_{V}, a_{B}, n, c, s\right\}$, the set of parameters to be varied.

The target result is encoded as a binary decision for each cell: presence or absence of $c B R A$ expression indicating the site of primitive streak formation. We therefore define

$$
D_{i}=\left\{\begin{aligned}
1, & \text { where streak is hypothesized in cell } i, \\
-1, & \text { where no streak is hypothesized in cell } i .
\end{aligned}\right.
$$

Then the 'likelihood' of parameters $\theta$ can be calculated as

$$
\mathcal{L}_{i}(\theta) \sim \frac{1}{2}\left[1+\tanh \left(\frac{D_{i} f_{i}(\theta)}{\Delta}\right)\right],
$$

in cell $i$, which approximates a step function as $\Delta \rightarrow 0$ (Fig. S5). For all parameter searches, we use $\Delta=0.05$. The likelihood is calculated individually for each cell of each experimental design given to the algorithm. The product of the likelihoods (across cells, designs and parameters) is calculated giving the total likelihood for a given set of parameter values. The parameters used to calculate the total likelihood include all model parameters and the bead parameters relevant for the experiment. Only cells in the anterior half of the embryo are used to calculate the total likelihood, because beads are only grafted anteriorly in the experiments modeled. As a result of this, Model B does not always predict the presence of an endogenous streak next to the posterior margin.

The posterior distributions of the parameters were obtained using MCMC Bayesian computation with the pyDREAM package (47) which implements a DREAM DSS $_{(\mathrm{Z})}$ algorithm (48). The algorithm was run using 5 Markov chains for a minimum of 5000 
iterations per chain, and convergence was tested using the Gelman-Rubin statistic (49, 50). The posterior distributions are shown in Figure S6.

The parameter search generates sets of parameters and corresponding likelihood values obtained through the Bayesian parameter estimation. In addition to calculating the likelihood, we assess whether each set of parameters predicts the correct number and position of streaks to replicate the target result. This allows us to define 'success' or 'failure' for each model and for each experimental design. The parameter values used in the plots in Figures 3-6 and S3 were chosen to maximize both the success rate and the likelihood. All parameter values are given in Data S1.

The parameter search is performed for each group of experimental designs comprising Figures 1, 2, 4/5 and 6. Ideally, the parameter search must output a single set of bead parameters, allowing both models to approximate the target results as closely as possible (Fig. $3 \mathrm{H}$ ). However, this acts as a restriction that might limit the ability of either model to replicate the target result. Therefore, the parameter search was also performed with all parameters varying for both models independently removing this restriction (Fig. $3 \mathrm{I}$ ). We verified that seeking a single set of bead parameters did not reduce the ability of either model to replicate the target result (Fig. $3 \mathrm{~J}$ ).

\section{References.}

1. L. Wolpert, in Towards a Theoretical Biology, C. H. Waddington, Ed. (Aldine Publishing Co., 1968), vol. 1.

2. L. Wolpert, Positional information and the spatial pattern of cellular differentiation. Journal of Theoretical Biology 25, 1-47 (1969).

3. J. Sharpe, Wolpert's French Flag: what's the problem? Development 146, (2019).

4. J. B. Green, J. C. Smith, Graded changes in dose of a Xenopus activin A homologue elicit stepwise transitions in embryonic cell fate. Nature 347, 391-394 (1990).

5. H. Bode, Axis formation in hydra. Annu Rev Genet 45, 105-117 (2011).

6. H. C. Schaller, Isolation and characterization of a low-molecular-weight substance activating head and bud formation in hydra. J Embryol Exp Morphol 29, 27-38 (1973).

7. S. B. Shah et al., Misexpression of chick Vg1 in the marginal zone induces primitive streak formation. Development 124, 5127-5138 (1997).

8. A. Streit et al., Chordin regulates primitive streak development and the stability of induced neural cells, but is not sufficient for neural induction in the chick embryo. Development 125, 507-519 (1998).

9. F. Bertocchini, C. D. Stern, Gata2 provides an early anterior bias and uncovers a global positioning system for polarity in the amniote embryo. Development 139, 4232-4238 (2012).

10. A. Streit, C. D. Stern, Mesoderm patterning and somite formation during node regression: differential effects of chordin and noggin. Mech Dev 85, 85-96 (1999).

11. F. Bertocchini, C. D. Stern, The hypoblast of the chick embryo positions the primitive streak by antagonizing nodal signaling. Dev Cell 3, 735-744. (2002).

12. I. Skromne, C. D. Stern, A hierarchy of gene expression accompanying induction of the primitive streak by Vg1 in the chick embryo. Mech Dev 114, 115-118 (2002). 
13. F. Bertocchini, I. Skromne, L. Wolpert, C. D. Stern, Determination of embryonic polarity in a regulative system: evidence for endogenous inhibitors acting sequentially during primitive streak formation in the chick embryo. Development 131, 3381-3390 (2004).

14. A. Torlopp et al., The transcription factor Pitx 2 positions the embryonic axis and regulates twinning. Elife 3, e03743 (2014).

15. E. Mitrani et al., Activin can induce the formation of axial structures and is expressed in the hypoblast of the chick. Cell 63, 495-501 (1990).

16. C. D. Stern et al., Activin and its receptors during gastrulation and the later phases of mesoderm development in the chick embryo. Dev Biol 172, 192-205. (1995).

17. P. B. Yu et al., Dorsomorphin inhibits BMP signals required for embryogenesis and iron metabolism. Nat Chem Biol 4, 33-41 (2008).

18. C. F. Arias, M. A. Herrero, C. D. Stern, F. Bertocchini, A molecular mechanism of symmetry breaking in the early chick embryo. Sci Rep 7, 15776 (2017).

19. H. C. Lee et al., Molecular anatomy of the pre-primitive-streak chick embryo. Open Biol 10, 190299 (2020).

20. F. Crick, Diffusion in embryogenesis. Nature 225, 420-422 (1970).

21. J. Miller, S. E. Fraser, D. McClay, Dynamics of thin filopodia during sea urchin gastrulation. Development 121, 2501-2511 (1995).

22. F. A. Ramirez-Weber, T. B. Kornberg, Cytonemes: cellular processes that project to the principal signaling center in Drosophila imaginal discs. Cell 97, 599-607 (1999).

23. S. Roy, F. Hsiung, T. B. Kornberg, Specificity of Drosophila cytonemes for distinct signaling pathways. Science 332, 354-358 (2011).

24. T. Gustafson, L. Wolpert, Studies on the cellular basis of morphogenesis in the sea urchin embryo. Gastrulation in vegetalized larvae. Exp Cell Res 22, 437-449 (1961).

25. W. Driever, C. Nusslein-Volhard, The bicoid protein determines position in the Drosophila embryo in a concentration-dependent manner. Cell 54, 95-104 (1988).

26. T. Gregor, E. F. Wieschaus, A. P. McGregor, W. Bialek, D. W. Tank, Stability and nuclear dynamics of the bicoid morphogen gradient. Cell 130, 141-152 (2007).

27. J. B. Gurdon, P. Harger, A. Mitchell, P. Lemaire, Activin signalling and response to a morphogen gradient. Nature 371, 487-492 (1994).

28. J. B. Gurdon, A. Mitchell, D. Mahony, Direct and continuous assessment by cells of their position in a morphogen gradient. Nature 376, 520-521 (1995).

29. N. McDowell, A. M. Zorn, D. J. Crease, J. B. Gurdon, Activin has direct long-range signalling activity and can form a concentration gradient by diffusion. Curr Biol 7, 671681 (1997).

30. R. D. Riddle, R. L. Johnson, E. Laufer, C. Tabin, Sonic hedgehog mediates the polarizing activity of the ZPA. Cell 75, 1401-1416 (1993).

31. C. Tickle, D. Summerbell, L. Wolpert, Positional signalling and specification of digits in chick limb morphogenesis. Nature 254, 199-202 (1975).

32. E. Rozbicki et al., Myosin-II-mediated cell shape changes and cell intercalation contribute to primitive streak formation. Nat Cell Biol 17, 397-408 (2015).

33. O. Voiculescu, F. Bertocchini, L. Wolpert, R. E. Keller, C. D. Stern, The amniote primitive streak is defined by epithelial cell intercalation before gastrulation. Nature 449, 1049-1052 (2007).

34. O. Voiculescu, L. Bodenstein, I. J. Lau, C. D. Stern, Local cell interactions and selfamplifying individual cell ingression drive amniote gastrulation. Elife 3, e01817 (2014). 
35. A. Perea-Gomez et al., Nodal antagonists in the anterior visceral endoderm prevent the formation of multiple primitive streaks. Dev Cell 3, 745-756 (2002).

36. J. Cotterell, A. Robert-Moreno, J. Sharpe, A Local, Self-Organizing Reaction-Diffusion Model Can Explain Somite Patterning in Embryos. Cell Syst 1, 257-269 (2015).

37. C. A. Pannett, A. Compton, The cultivation of tissues in saline embryonic juice. Lancet $\mathbf{1}$, 381-384 (1924).

38. D. A. T. New, A new technique for the cultivation of the chick embryo in vitro. $J$. Embryol. exp. Morph. 3, 326-331 (1955).

39. C. D. Stern, G. W. Ireland, An integrated experimental study of endoderm formation in avian embryos. Anat Embryol 163, 245-263 (1981).

40. C. D. Stern, Detection of multiple gene products simultaneously by in situ hybridization and immunohistochemistry in whole mounts of avian embryos. Curr Top Dev Biol 36, 223-243 (1998).

41. A. Streit, C. D. Stern, Combined whole-mount in situ hybridization and immunohistochemistry in avian embryos. Methods 23, 339-344. (2001).

42. A. Kispert, H. Ortner, J. Cooke, B. G. Herrmann, The chick Brachyury gene: developmental expression pattern and response to axial induction by localized activin. Dev Biol 168, 406-415 (1995).

43. K. F. Liem, Jr., G. Tremml, H. Roelink, T. M. Jessell, Dorsal differentiation of neural plate cells induced by BMP-mediated signals from epidermal ectoderm. Cell 82, 969-979 (1995).

44. C. Papanayotou et al., Calfacilitin is a calcium channel modulator essential for initiation of neural plate development. Nat Commun 4, 1837 (2013).

45. M. E. Dickinson et al., Chromosomal localization of seven members of the murine TGFbeta superfamily suggests close linkage to several morphogenetic mutant loci. Genomics 6, 505-520 (1990).

46. A. F. Candia et al., Cellular interpretation of multiple TGF-beta signals: intracellular antagonism between activin/BVg1 and BMP-2/4 signaling mediated by Smads. Development 124, 4467-4480 (1997).

47. E. M. Shockley, J. A. Vrugt, C. F. Lopez, PyDREAM: high-dimensional parameter inference for biological models in python. Bioinformatics 34, 695-697 (2017).

48. E. Laloy, J. A. Vrugt, High-dimensional posterior exploration of hydrologic models using multiple-try DREAM(ZS) and high-performance computing. Water Resources Research 48, (2012).

49. A. Gelman, D. B. Rubin, Inference from Iterative Simulation Using Multiple Sequences. Statistical Science 7, 457-472 (1992).

50. S. P. Brooks, A. Gelman, General Methods for Monitoring Convergence of Iterative Simulations. Journal of Computational and Graphical Statistics 7, 434-455 (1998).

Funding: This research was supported by Basic Science Research Program through the National Research Foundation of Korea (NRF) funded by the Ministry of Education (2014R1A6A3A03053468) to HCL, by an MRC DTP studentship (MR/N013867/1) to $\mathrm{CH}$ and by a Wellcome Trust Investigator Award (107055/Z/15/Z) to CDS. 
Author contributions: HCL conducted all embryo experiments; $\mathrm{CH}$ designed the models and implemented them; NMMO constructed the vectors and performed cell culture; RPC and KP provided advice and ideas on mathematical methods; LW provided inspiration and stimulated questions during the early stages of the study; CDS supervised the study. HCL, CH and CDS wrote the paper.

Competing interests: The authors declare no competing interests.

Data and materials availability: The software used for the mathematical and computational modelling is available at https://github.com/catohaste/neighbourhoodstreak. 

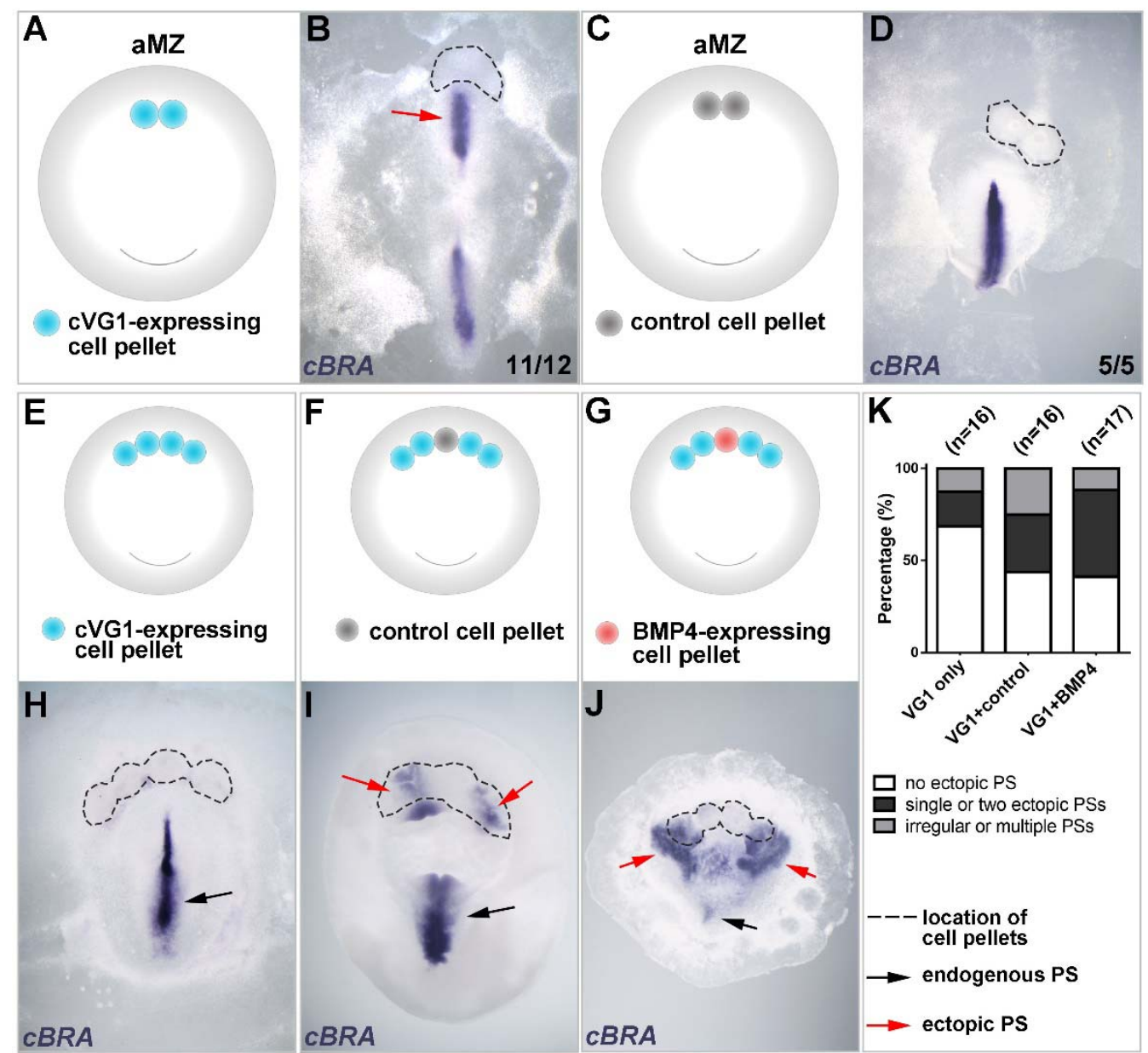

Fig. 1. Interruption of a domain exposed to an inducing signal increases the incidence of primitive streak induction - experiments with secreting cells. (A, B) When two pellets of cVG1-expressing cells are grafted in the anterior marginal zone (aMZ), only a single ectopic primitive streak (red arrow) is generated. (C, D) Control cell pellets do not induce a streak. The number of embryos displaying the phenotypes and the total is indicated in the lower right of each panel. (E-G) Experimental design. Ectopic streak formation is checked in three different conditions: misexpression of cVG1 in a wide area using four cVG1-expressing cell pellets (E), introduction of a 'spacer' (control cell pellet) to interrupt a set of four cVg1 pellets (F), and introduction of an inhibitor (BMP4-expressing cell pellet) to interrupt a set of four $\mathrm{cVg} 1$ inducing pellets $(\mathbf{G})$. (H-J) representative embryos for each experiment. The frequency of primitive streak formation is enhanced by interrupting the domain of inducing signal, even when this interruption is achieved by introduction of an inhibitor $(\mathbf{J})$. (K) Summary graph showing the incidence of each type of result for the above experiments (E-J). PS: primitive streak. Black and red arrows, endogenous and ectopic streaks, respectively. Dotted lines, position of the cell pellets. $c B R A$, primitive streak marker. 


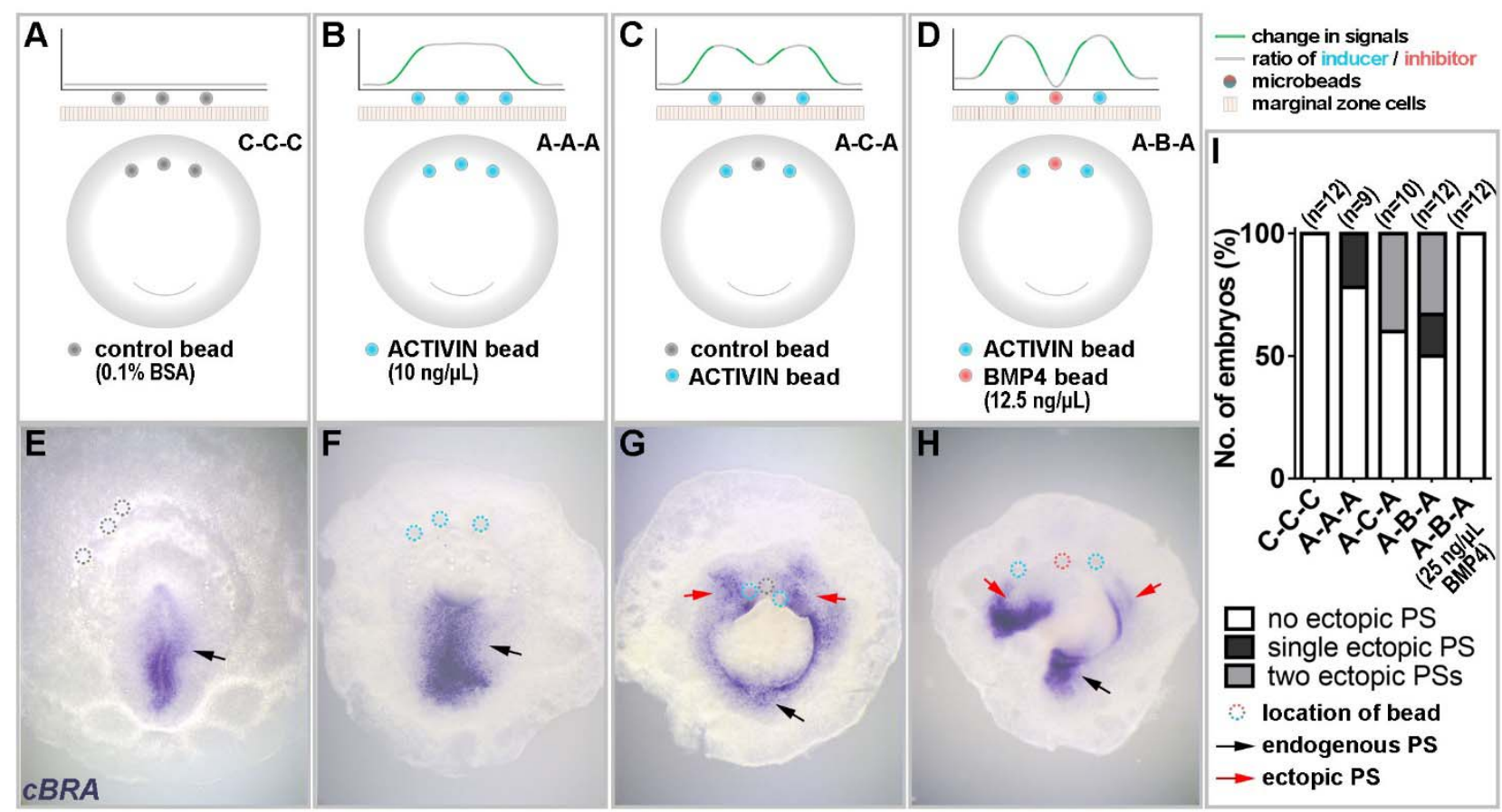

Fig. 2. Interruption of a domain exposed to an inducing signal increases the incidence of primitive streak induction - experiments with protein-soaked beads. (A-D) Experimental design. Induction of a streak is assessed after four combinations of bead grafts: 3 control beads (A), exposing a wide area to the inducing signal by grafting 3 ACTIVIN-soaked beads (B), interrupting the inducing signal by adding a 'space' (control bead) to separate two adjacent inducing (ACTIVIN) beads (C), and adding an inhibitor (BMP4-soaked bead) to separate two adjacent ACTIVIN beads (D). (E-H) Representative embryos for each experiment. Two primitive streaks only form when the inducing signal is interrupted, even when adding an inhibitory signal. (I) Summary graph showing the incidence of each type of result for the above experiments. Note that a higher concentration of BMP4 $(25 \mathrm{ng} / \mu \mathrm{l})$, does not allow an ectopic streak to form. Dotted circles, location of beads. Other abbreviations and symbols as in Fig. 1. 

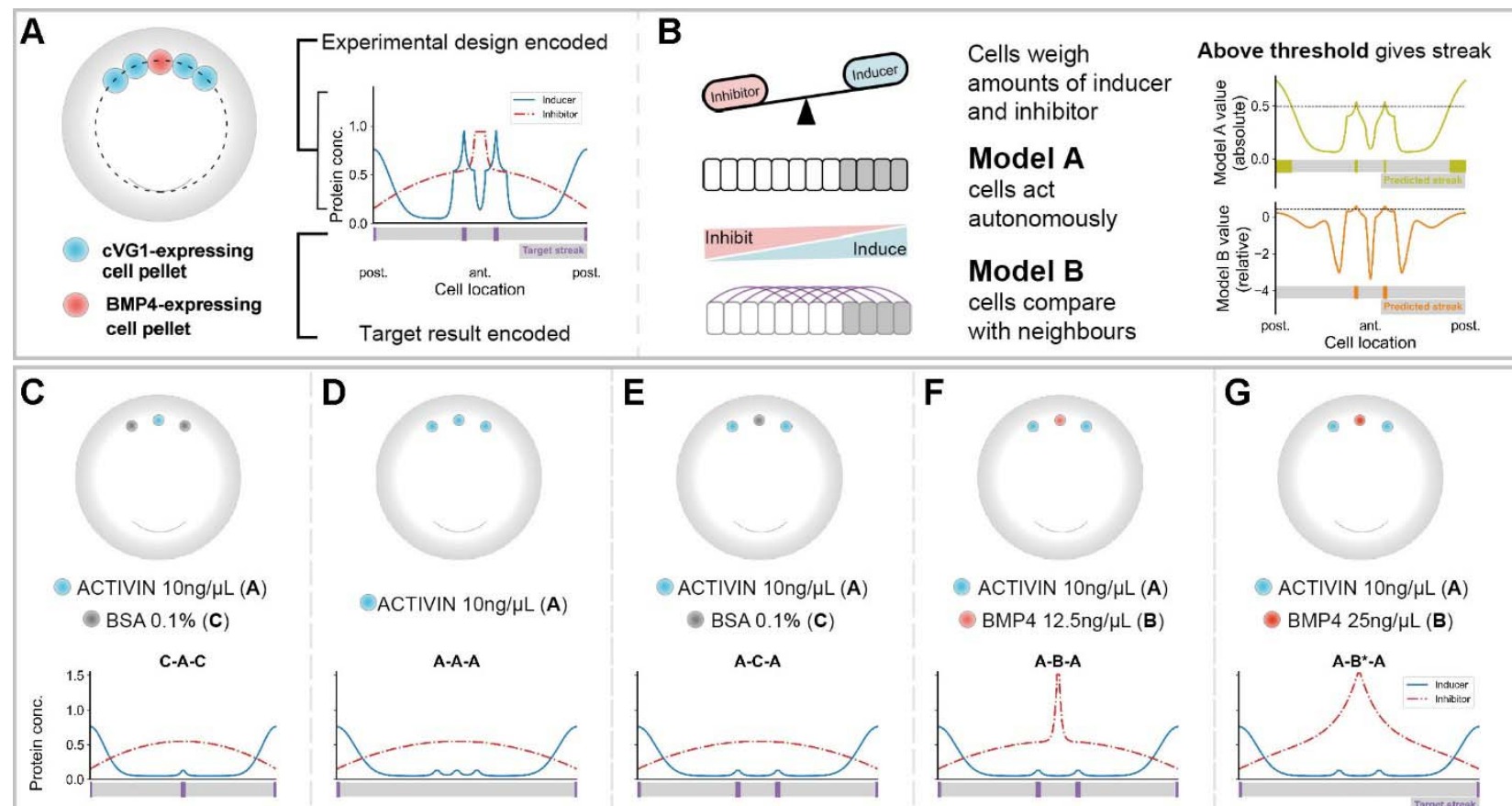

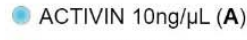

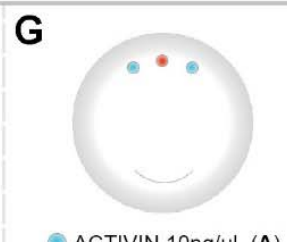

A-C-A
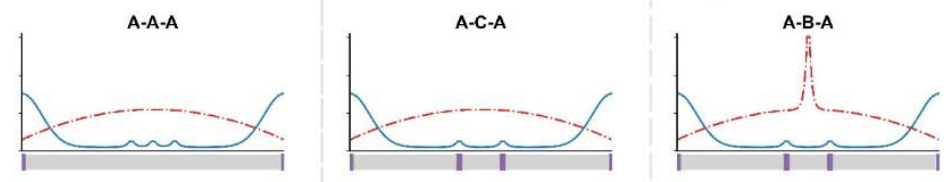
- BMP4 25ng/ $\mu \mathrm{L}$ (B)
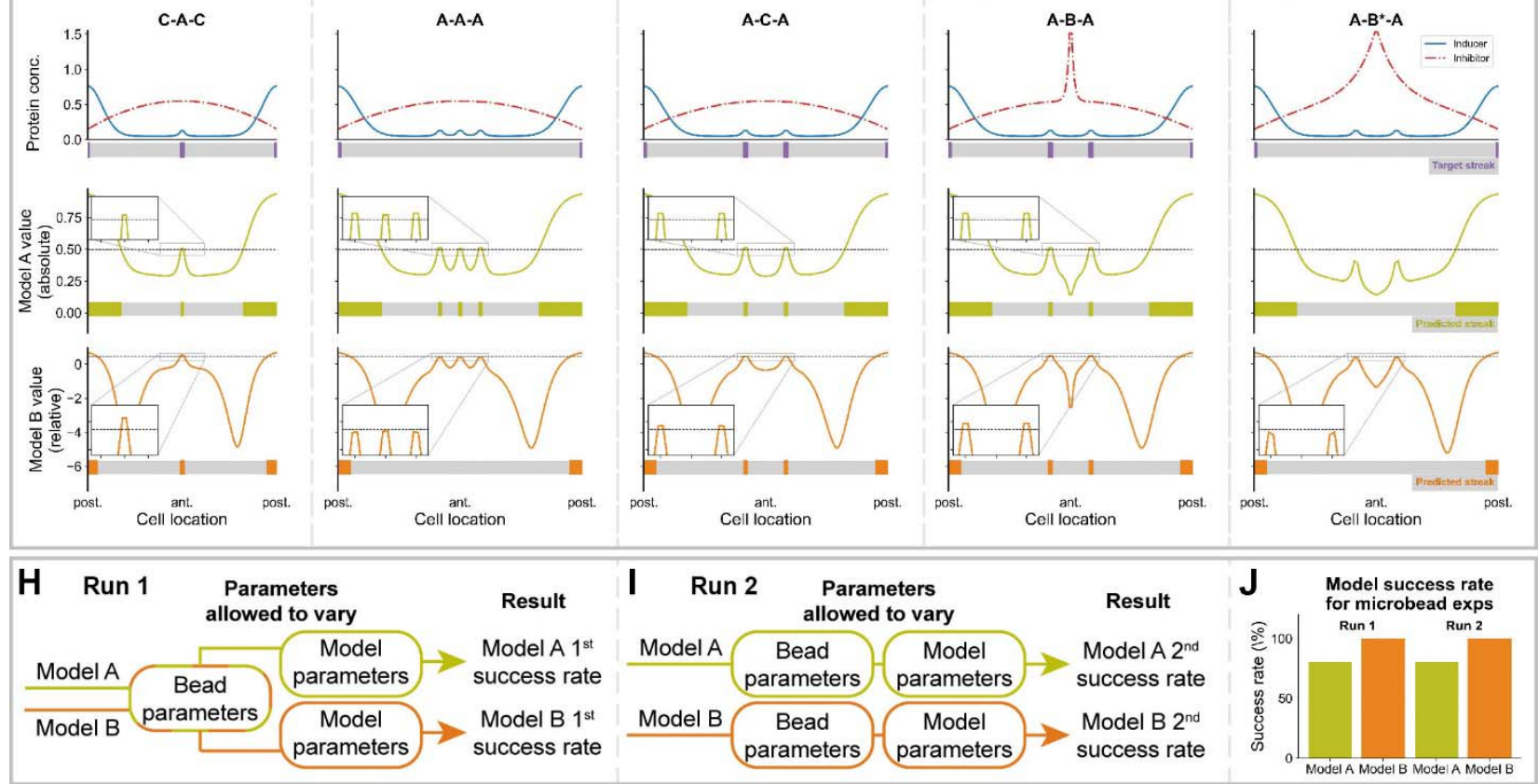

Fig. 3. Mathematical model and verification in silico. (A-B) Model workflow. (A) The dotted line represents the marginal zone. Concentrations of primitive-streak-inducing and -inhibiting proteins are inferred from experimental design. Target site of streak initiation is encoded for comparison with model predictions. (B) In each cell, both models weigh concentrations of streak-inducing and -inhibiting proteins. Model A assumes that cells act autonomously to define the site of streak formation. Model B assumes that cells compare concentrations within a given neighborhood to initiate streak formation. Model values are plotted for the entire embryo, where values above a threshold define the site of streak initiation. (C-G) In silico simulations of bead experiments in Figure 2. Top, experimental designs. First row of plots: inducer levels shown as a red line, inhibitor in blue; the lower bar marks the expected position of streak initiation. Second row of plots: Model A values and corresponding predicted streak locations. Third row: Model B values and streak locations. (C, E-G) A model is defined as "successful" for one experimental design if the predicted number and location of streaks matches the target result. (D) Model A fails to replicate the experimental result. No parameter values are found where Model A is 
successful for both designs (C) and (D). (E-F) Unlike Model A, Model B predicts that exchanging the control bead for a bead of inhibitor will counter-intuitively increase the chances of ectopic streak formation (insets). (H-J) To ensure that finding a single set of parameters does not limit the ability of either model to replicate the target results, we used two approaches for parameter estimation: (H) a single set of bead parameters is defined for both models, or (I) bead and model parameters vary freely for both models, allowing the maximum chance of success. (J) approach $\mathrm{H}$ does not reduce the success rate of either model. Model B outperforms Model A in all cases. 

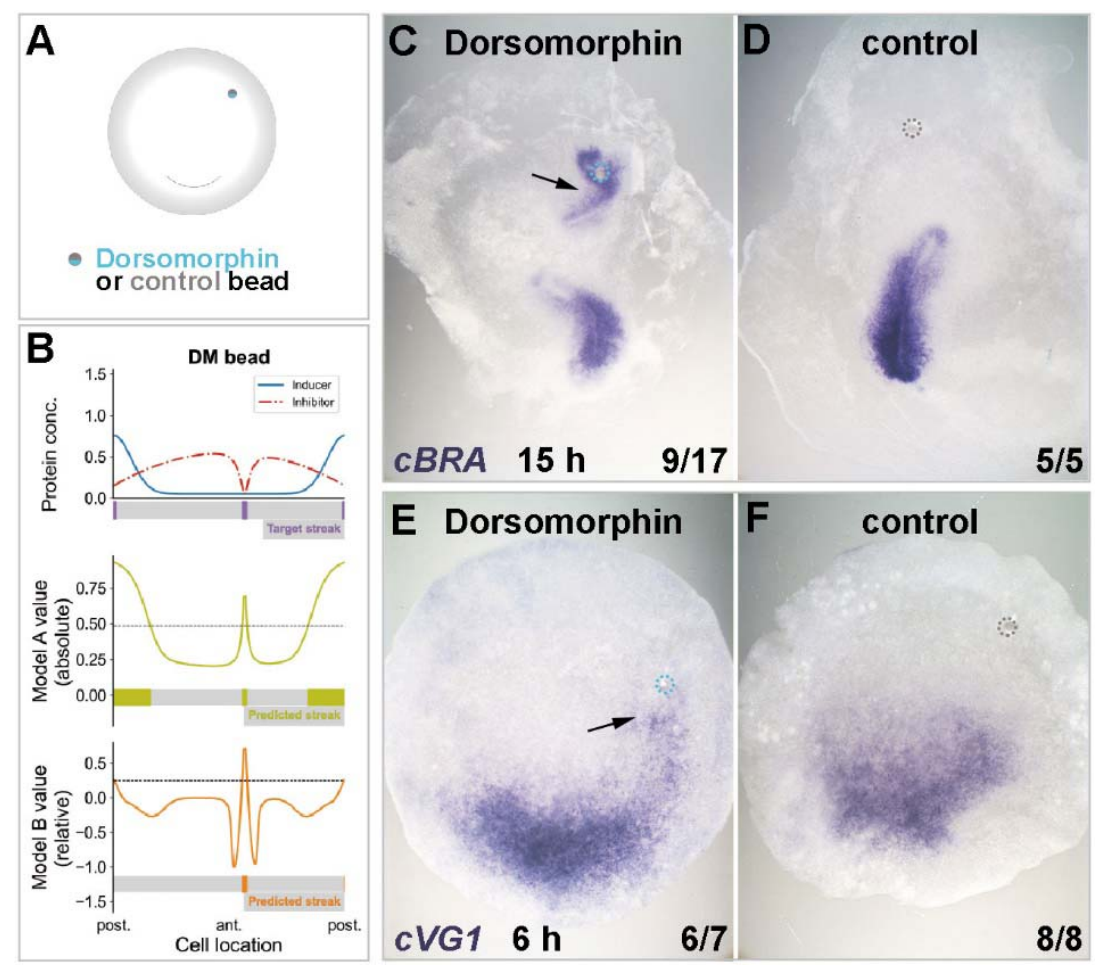

Fig. 4. Decreasing the amount of inhibitor induces ectopic primitive streak formation. Local repression of inhibitor (BMP) using Dorsomorphin induces a streak both in silico and in vivo. (A) Experimental setup. (B) Results of in silico simulations (colors and other conventions as in Fig. 3). Both models predict ectopic primitive streak formation when the concentration of inhibitor is decreased locally. (C-F) Results of in vivo experiments. A graft of a $1 \mathrm{mM}$ Dorsomorphin-soaked bead in the anterior marginal zone induces formation of an ectopic streak expressing $c B R A$ after overnight culture $(\mathbf{C}$, arrow), which is preceded (at $6 \mathrm{~h}$ ) by ectopic expression of $c V G 1$ (E, arrow). Control ( $0.2 \%$ DMSO) beads have no effect (D, F). Dotted circles, location of microbeads. The proportion of embryos showing the phenotype illustrated are indicated in the lower right of each panel. 

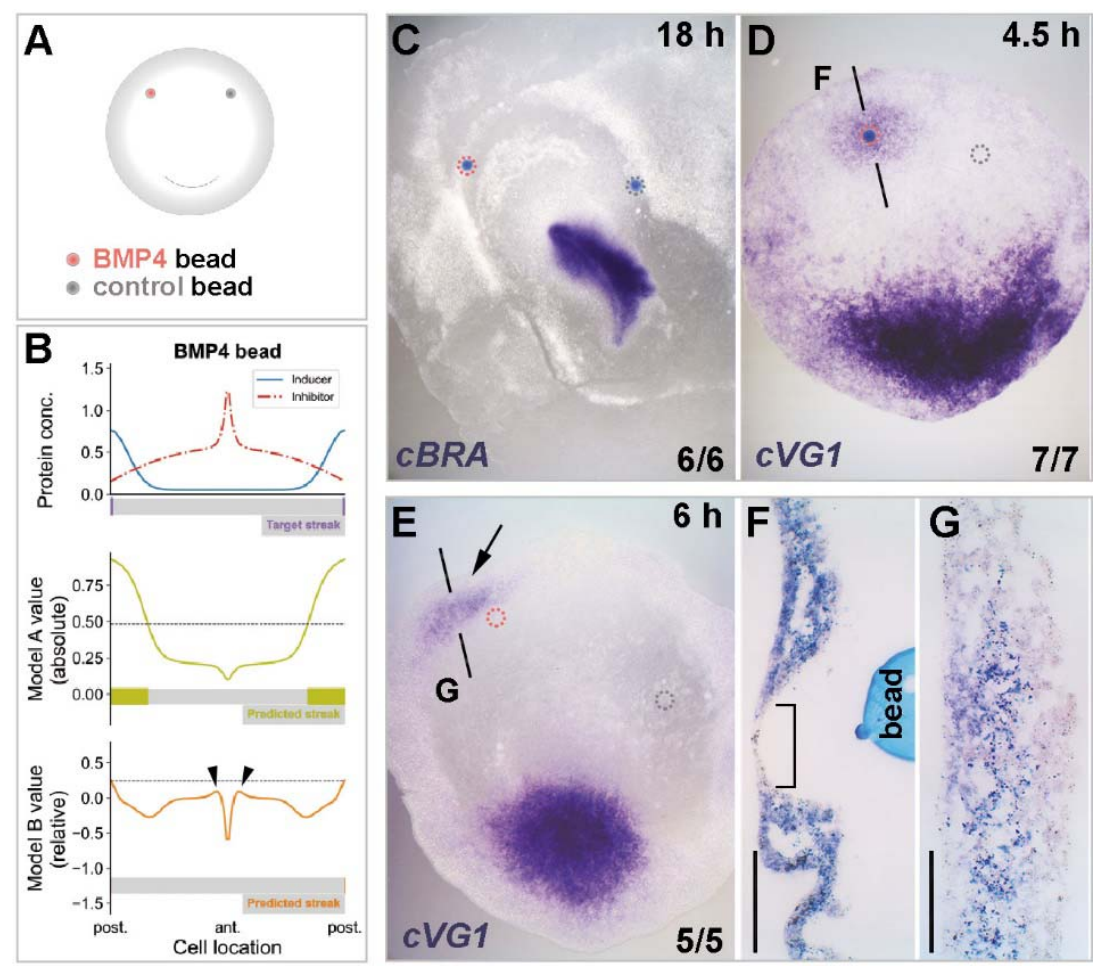

Fig. 5. Increasing the amount of inhibitor augments the streak-inducer. Local overexpression of inhibitor (BMP4) increases streak-inducing values in silico, and cVG1 expression in vivo in neighboring cells. (A) Experimental setup. (B) Results of in silico simulations. Only Model B predicts an increase in streak-inducing value in cells neighboring the bead of inhibitor (arrowheads), but at levels insufficient to initiate an ectopic streak. (C-G) Results of in vivo experiments. No ectopic primitive streak (marked by $c B R A$ ) is induced overnight after a graft of BMP4 $(50 \mathrm{ng} / \mu \mathrm{l})$ soaked bead $(\mathbf{C})$. However, a short time $(4.5 \mathrm{~h})$ after grafting, ectopic $c V G 1$ expression is induced in the marginal zone (D) in neighboring cells (F) but not in the cells lying directly above the bead (F, square bracket). By $6 \mathrm{~h}$ after grafting, induced $c V G 1$ expression is no longer visible in the marginal zone, remaining only in the extraembryonic endoderm (germ wall) (E) arrow and $\mathbf{G})$. The dashed lines in (D and E) indicate the level of the sections in (F and $\mathbf{G})$. Dotted circles, location of microbeads. The proportion of embryos showing the illustrated phenotypes is indicated on the lower right of each panel. Scale bar for $(\mathbf{F}$ and $\mathbf{G}), 100 \mu \mathrm{m}$. 


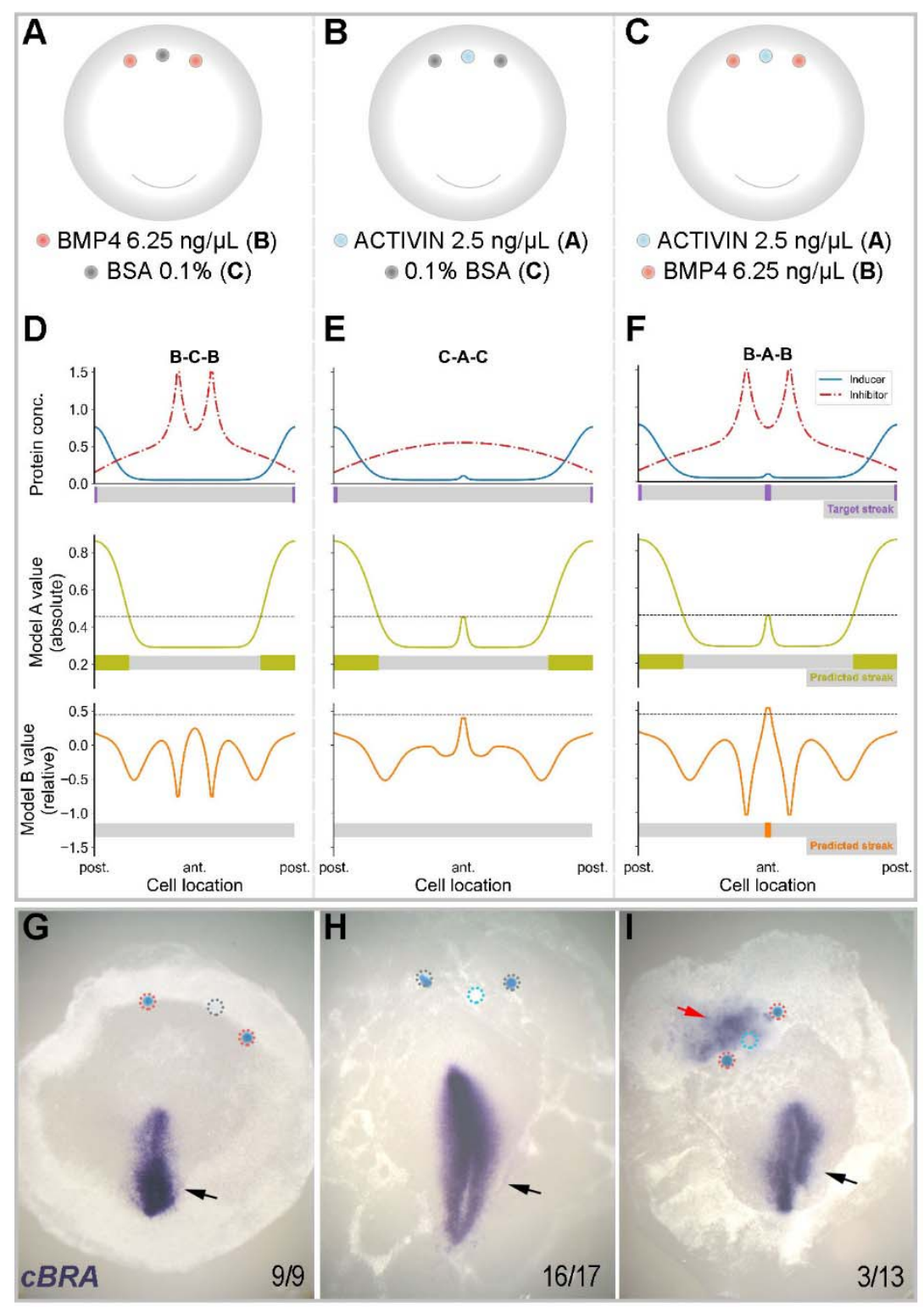

Fig. 6. Challenging the models: effect of placing an inhibitor next to sub-threshold amounts of inducer. (A-C) Experimental design. Three conditions were tested: two BMP4 beads $(6.25$ $\mathrm{ng} / \mu \mathrm{l})(\mathrm{B})$ separated by a control bead (C) (A), a bead loaded with sub-threshold $(2.5 \mathrm{ng} / \mu \mathrm{l})$ amounts of ACTIVIN (A) flanked by two control beads (C) (B) and a sub-threshold bead of activin flanked by two beads of inhibitor (BMP4) (C). (D-F) Results of in silico simulations. Only Model B predicts that introducing a sub-threshold amount of inducer flanked by beads of inhibitor will paradoxically generate a site of ectopic PS formation. (G-I) Results of in vitro experiments showing representative embryos for each experiment. Number of embryos showing the phenotypes are indicated in each panel. In vivo, grafting a sub-threshold ACTIVIN bead flanked by two BMP4 beads in the marginal zone can induce ectopic $c B R A$ expression (I). No such induction is seen in the other combinations (B-C-B or C-A-C) $(\mathbf{A}, \mathbf{B}, \mathbf{G}, \mathbf{H})$. Black and red arrows: endogenous and ectopic $c B R A$ expression, respectively. Dotted circles: location of microbeads. The numbers on the lower right of panels G-I indicate the frequency of the illustrated result for each experimental combination. 


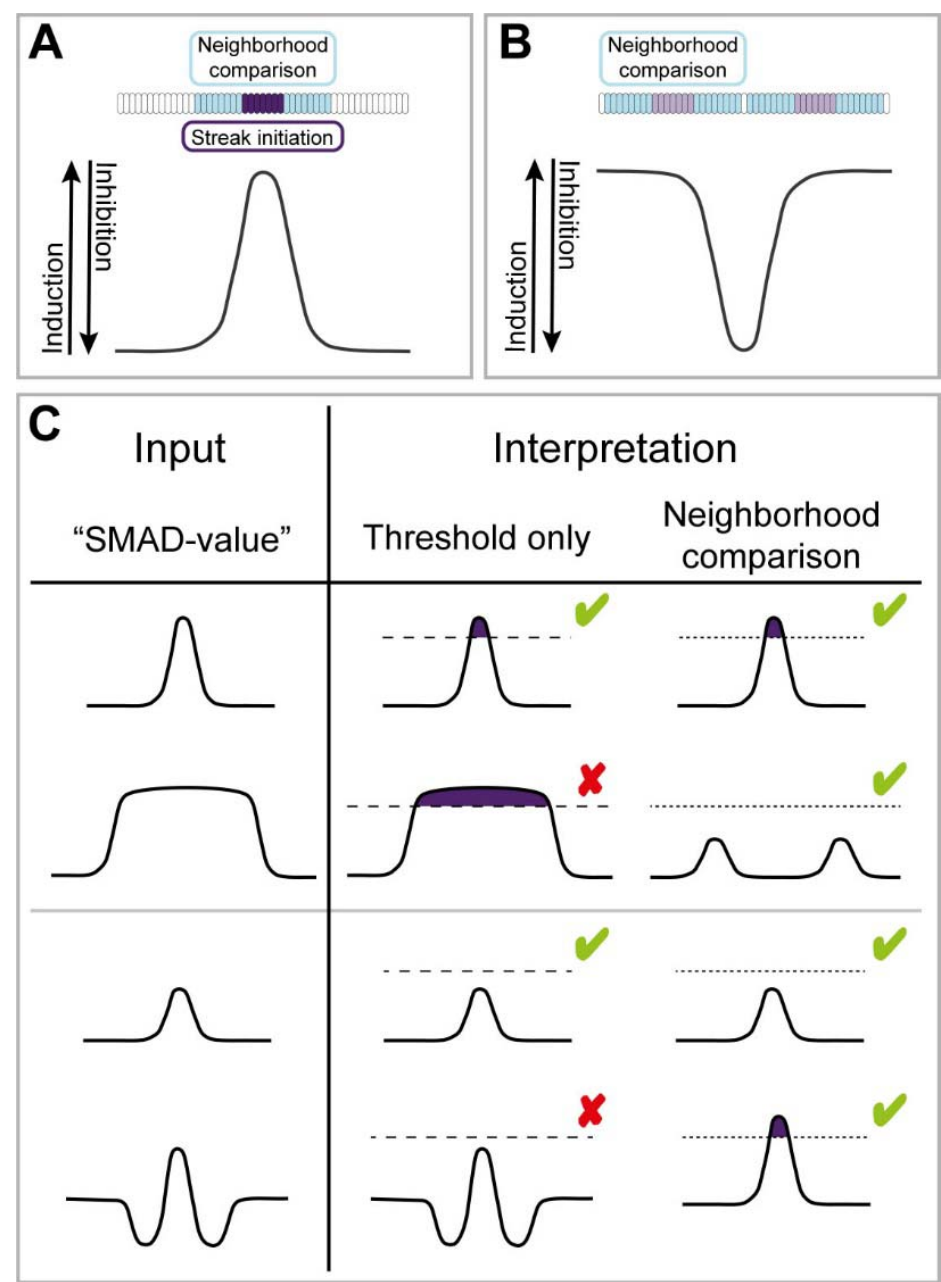

Fig. 7. A "neighborhood watch" model accounts for positioning the site where primitive streak formation is initiated in the marginal zone of the early chick embryo. (A-B) The "SMAD-value" represents a combination of inducing and inhibiting signals. Cells assess their position by comparing their SMAD-value with those of their neighbors. Blue: territory over which cells are able to sense. Purple: cell(s) initiating primitive streak formation. Light purple: partial/weak induction. (A) The domain of induction must be sufficiently narrow for cells to sense a local maximum. When a local maximum is located, primitive streak formation is initiated in the marginal zone. (B) Cells adjacent to a domain of inhibition detect their relatively high SMAD-value and react by emitting streak-inducing signals (cVG1). However, the induction is not sufficiently strong to initiate the formation of a full streak (no $c B R A$ expression). (C) Comparison of predictions by two models: one ("threshold only") where positional information is interpreted cell-autonomously solely by assessing the morphogen concentrations, and another ("neighborhood watch") where cells make local comparisons with their neighbors to assess their position in the gradients. First row: a narrow domain of induction results in initiation of primitive streak formation. Second row: broadening the domain of induction distinguishes between the two models. The "neighborhood watch" model predicts that streak formation will not be initiated, matching experimental data. Third row: a sub-threshold amount of inducer results in no ectopic $c B R A$ expression. Fourth row, the "threshold only" model predicts that adding inhibitor adjacent to a sub-threshold amount of inducer will either have no effect or reduce the chance of ectopic streak formation. In contrast, the "neighborhood watch" 
model correctly predicts the counter-intuitive result that addition of inhibitor increases the chances of ectopic streak initiation. Green ticks and red crosses represent whether the model prediction matches the experimental data or not, respectively. Dashed and dotted lines represent thresholds for interpretation of morphogen concentration. Purple: primitive streak formation initiated in cells above threshold. 


\section{Supplementary Materials}

Fig. S1. Expression of $c V G 1$ and BMP4 in the pre-primitive-streak chick embryo. (A) $c V G 1$ and $B M P 4$ are expressed as opposing gradients $-c V G 1$ is expressed as a very steep gradient decreasing from the posterior marginal zone, and BMP4 forms a shallower gradient decreasing in a posterior direction. (B) Gaussian and parabolic functions are used to model the opposing gradients of inducer and inhibitor, relating to cVG1 and BMP4 respectively.
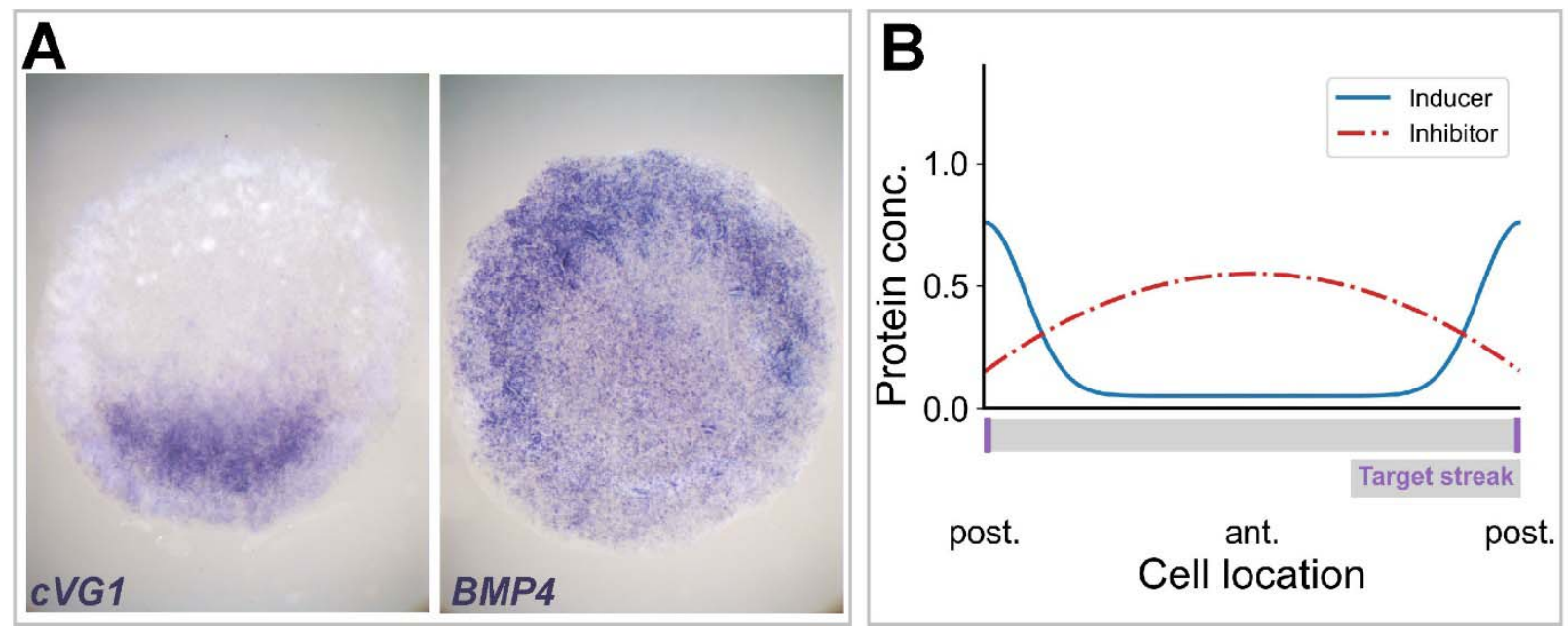
Fig. S2. Inducing or inhibitory effect of grafting ACTIVIN- or BMP4-soaked microbeads. (A-E) A graft of an ACTIVIN-soaked bead in the anterior marginal zone induces an ectopic primitive streak (arrow) at concentration of $10 \mathrm{ng} / \mu \mathrm{l}(\mathbf{B})$ and $5 \mathrm{ng} / \mu \mathrm{l}(\mathbf{C})$, but not at $2.5 \mathrm{ng} / \mu \mathrm{l}$ (D); E shows a control (0.1\% BSA-soaked bead). Dotted circles, position of the bead. The proportion of embryos showing the effect illustrated is indicated in each panel. $c B R A$ : primitive streak marker. (F-J) A graft of a BMP4-soaked bead in the posterior marginal zone inhibits streak formation. (J) summarizes the incidence of the various types of result.
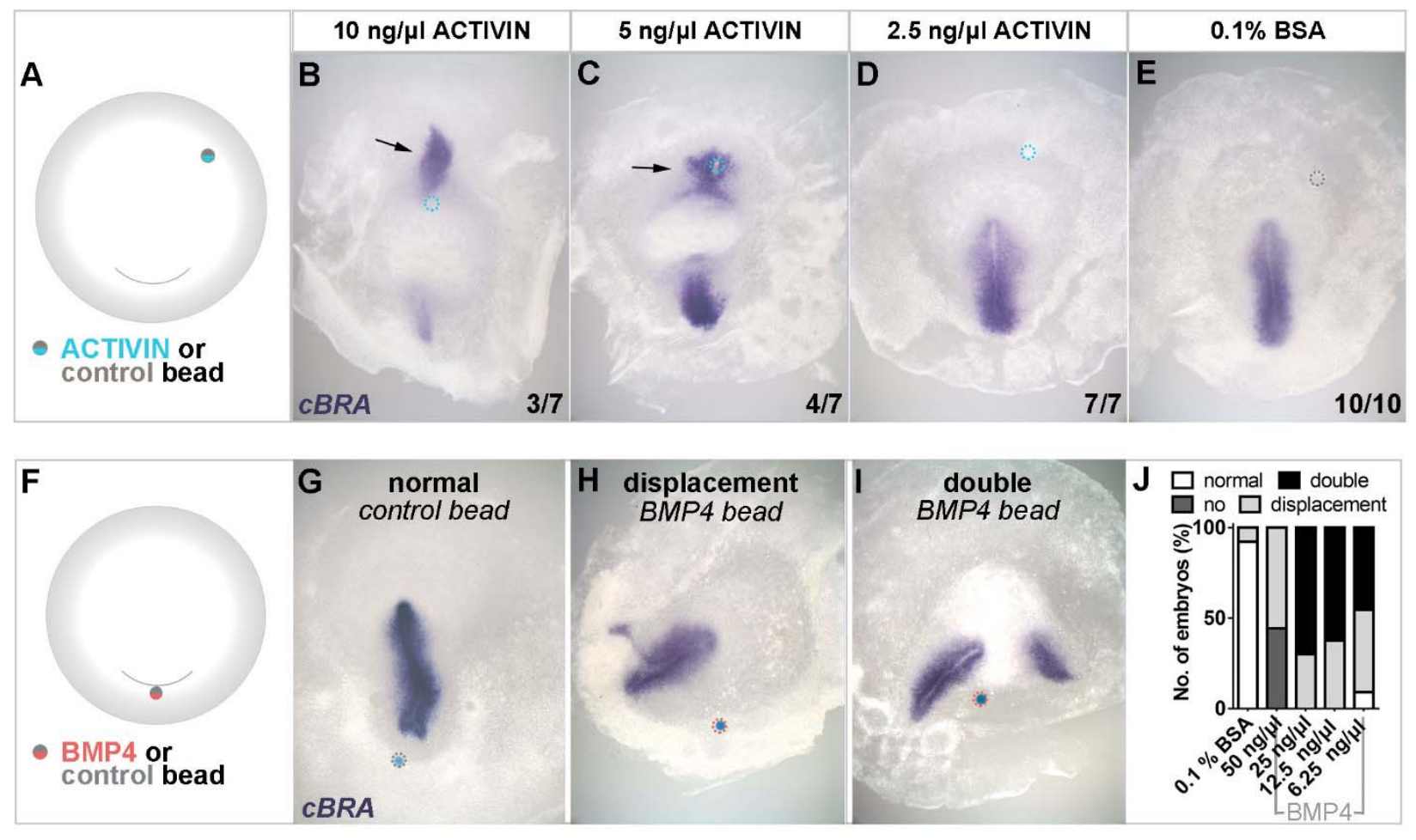
Fig. S3. Likelihood function used in Bayesian inference of parameters. The likelihood function is defined so that when a set of parameters allows a model prediction to mimic the target result, the value of the likelihood function is high (and vice-versa). The likelihood function approximates a step function.

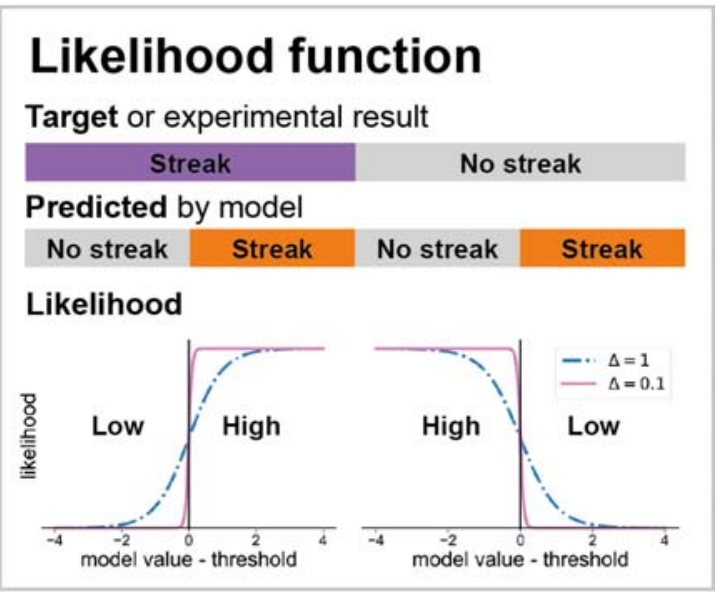


Fig. S4. In silico simulations of cell pellet experiments shown in Fig. 1. Top row: experimental designs. The first row of plots shows the encoded experimental design and the target result (based on the experimental findings). The second row of plots shows model A values and the corresponding predicted site of primitive streak initiation. The bottom row shows the results obtained with model B. (A-B) Model A predicts that broadening the domain of ectopic inducer increases the chance of ectopic $c B R A$ expression, whereas model $\mathrm{B}$ predicts that the occurrence of ectopic $c B R A$ expression will be reduced. The prediction of Model $\mathrm{B}$ aligns better with experimental results (Fig. 1 A-B, G, J, M). (C-D) Simulated results when a control or BMP4-expressing cell pellet is flanked by cVG1-expressing pellets.

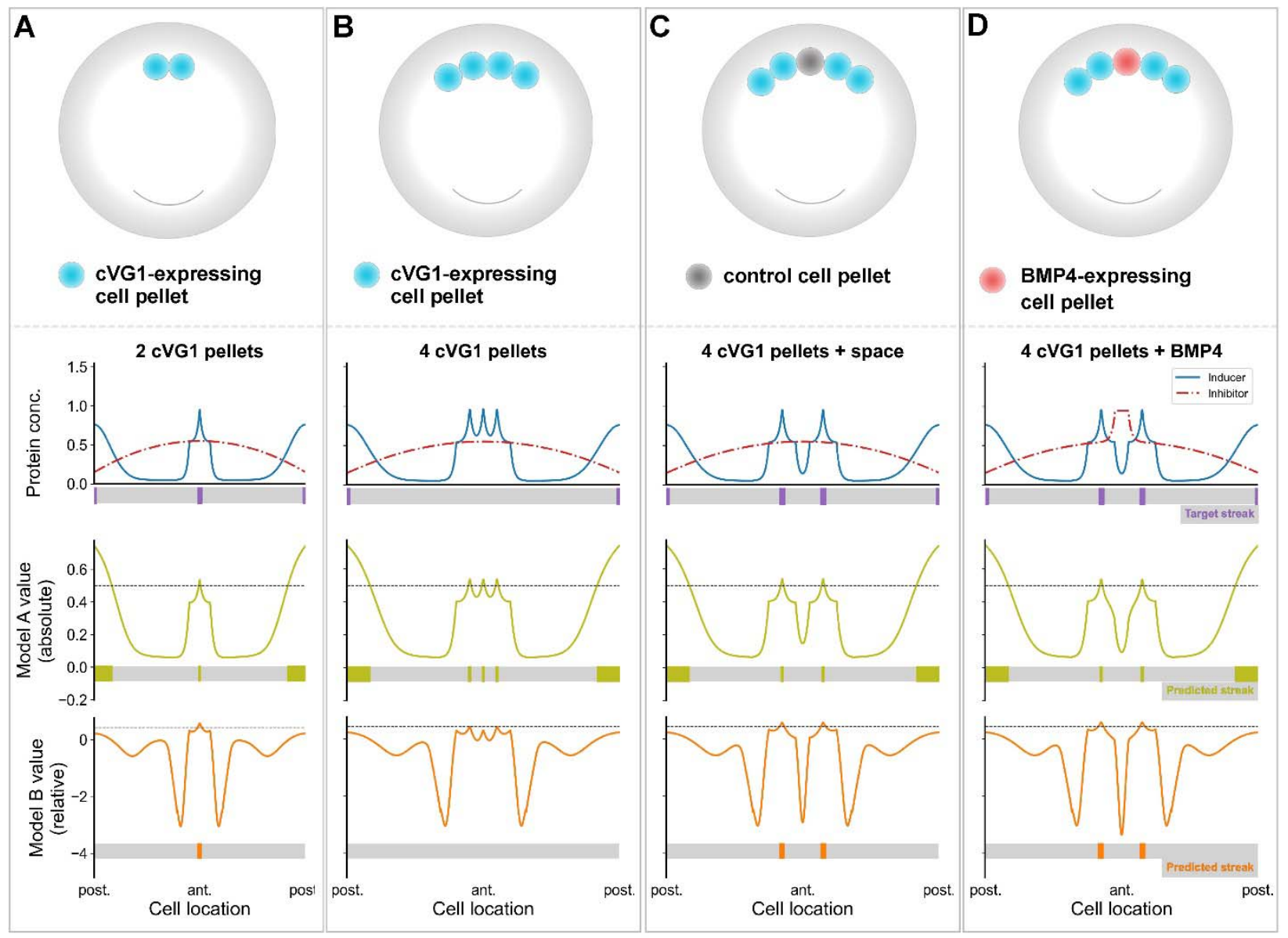


Fig. S5. Modeling the placement of beads. (A) The position of the bead relative to the embryo is encoded by the center of the bead and is governed by the experimental design. The placing of a bead causes a constant, additive change in protein concentration throughout the width of the bead. The magnitude of this change is defined as the concentration of the bound protein, $c$. The protein concentration then decays exponentially in space, at a rate governed by the spread parameter, $s$. During parameter estimation, the center and width of the bead are kept constant while the parameters $c$ and $s$ are permitted to vary. (B) Changing the concentration parameter $(c)$ principally changes the height of the peak (or trough) and has little effect on the number of cells affected by the bead placement. (C) Changing the spread parameter $(s)$ has no effect on the magnitude of protein concentration change, but can have a large impact on the size of the territory around the bead that is affected by the ligand.
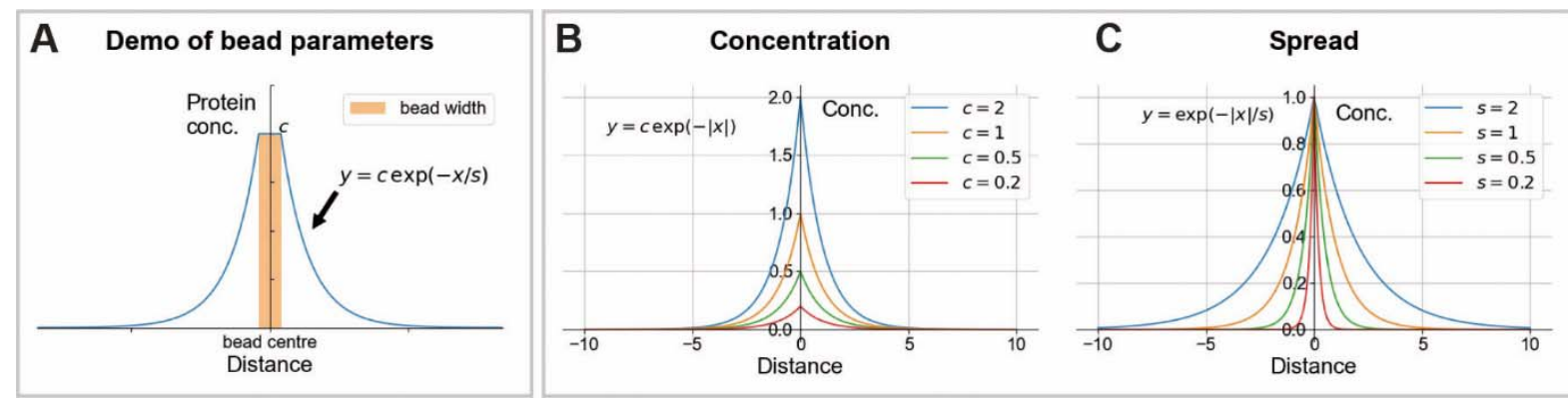
Fig. S6. Posterior distributions of parameters following Bayesian inference. Each cell of the table shows posterior distributions resulting from a single parameter search, corresponding to the figures shown. Separate distributions are given for models A and B, as the bead parameters were varied independently for the two models as described in Figure 3 I. In a single cell of the table, the plots along the leading diagonal give the marginal posterior distributions for each parameter. The plots in the top right corner and bottom left corner represent both represent joint distributions for two parameters, allowing the reading to study cross-talk between parameters. In the top right corner, joint distributions are represented with a scatter plot, where each point corresponds to a set of parameters tried during the parameter search and its color corresponds to the likelihood of this set of parameters. In the bottom left corner, joint distributions are represented by a contour plot where darker colors represent a higher density of parameter values sampled.

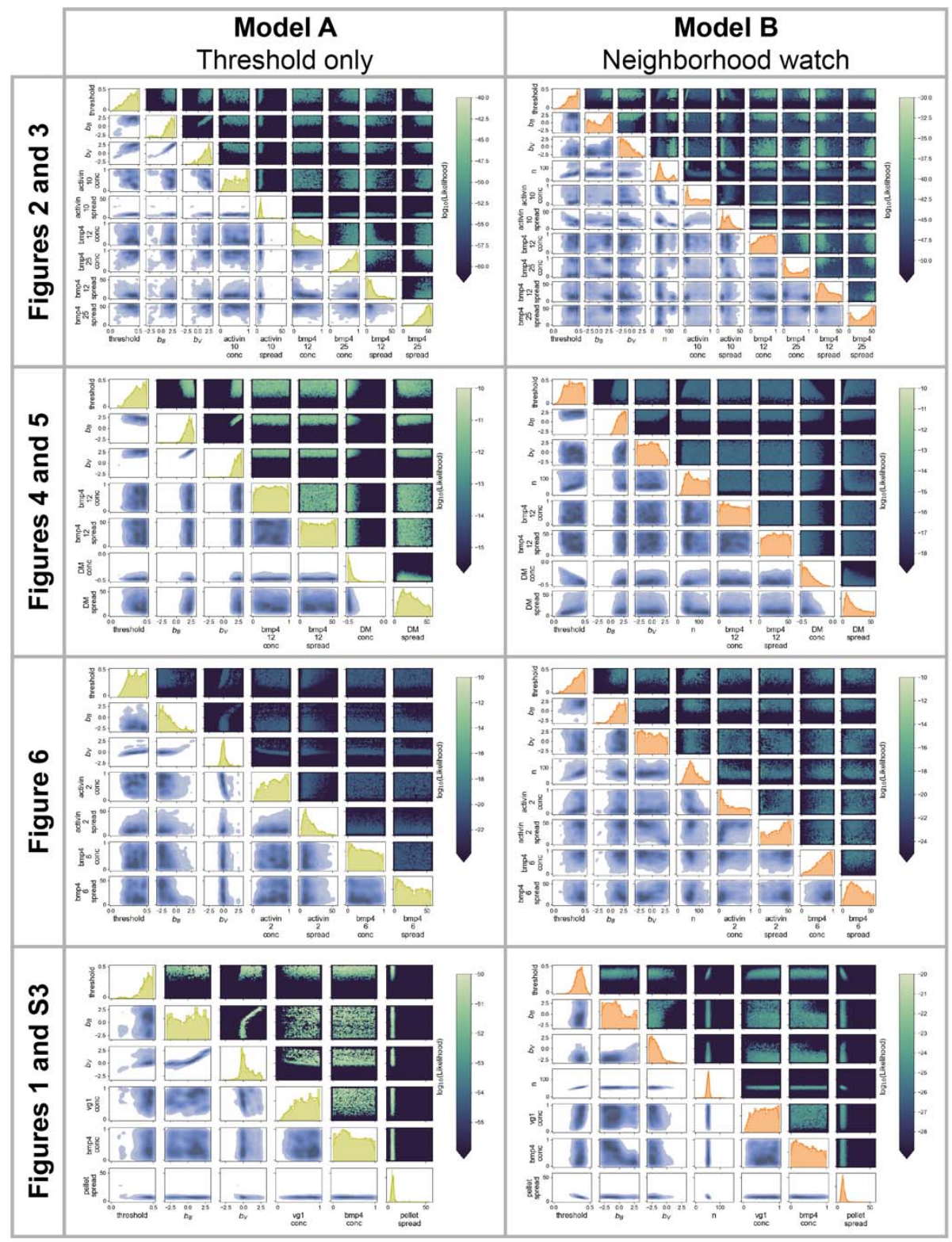


30 Data S1. Results of parameter searches. The parameter values giving the highest success rate for each parameter search are shown. Parameter searches were run separately for each group of experimental designs (rows 4-15). For each set of experimental designs, models A and B were run separately to give each model the best chance of predicting the experimental results accurately 34 (rows 4-5, 7-8, 10-11, 13-14). The parameter search was also run so as to predict a single set of 35 bead parameters for both models (rows 6, 9, 12, 15). The parameters given are for the pyDREAM 36 algorithm (columns D-F), for each model (columns G-M) and for the type of bead used (columns $37 \mathrm{~N}-\mathrm{AB}$ ). The result of each parameter search is denoted by the total likelihood (column AC), 38 success (TRUE) or failure (FALSE) of each experimental design (columns AD-BE) and the 39 overall success rate of each model (columns BF-BG). 